\title{
Zur Terminologie der Psychoanalyse [1973]
}

Die beiden germanistischen Arbeiten, die sich mit Freuds wissenschaftlichem Sprachstil beschäftigen, Walter Muschgs Essay über Sigmund Freud als Schriftsteller (1930) und die Abhandlung über Sigmund Freuds Prosa von Walter Schönau (1968), sind literaturwissenschaftlich orientiert und gehen nur am Rande auf die Terminologie Freuds und auf die in seiner Begriffssprache enthaltenen naturwissenschaftlichen Analogien ein. Dies beides steht im Mittelpunkt der folgenden sprachwissenschaftlichen Untersuchung. Die Arbeit wird erleichtert durch das Vokabular der Psychoanalyse von Laplanche und Pontalis (1972), das die Termini begrifflich klärt und kommentiert, sie in den Schriften Freuds und einiger seiner Schüler nachweist und auf ihre Herkunft und geschichtliche Entwicklung eingeht.

\section{Die wissenschaftliche Sprache Freuds und ihre Gründe}

Zunächst soll versucht werden, den Typus der von Freud bevorzugten Termini und die Art ihrer Verwendung zu charakterisieren und - soweit möglich - zu erklären.

Die allmähliche oder schubweise Entdeckung neuer Gegenstände und $\mathrm{Zu}$ sammenhänge ist ein allgemeines Kennzeichen der Wissenschaftsgeschichte. Dieser Erkenntnisfortschritt bedingt, allmählich oder schubweise, die Schaffung neuer Termini, die Ersetzung, Umdeutung und Erweiterung eingeführter Terminologien.

Auch Freud sah sich genötigt, das Neue an seinen Entdeckungen und Konzeptionen mit Hilfe eines spezifischen Vokabulars zu formulieren. Das Vokabular der Psychoanalyse erläutert insgesamt 331 Termini, die überwiegend von Freud stammen. Der Ausdruck Terminologie deckt sich in unserem Fall mit dem, was man als den Begriffsapparat der Psychoanalyse bezeichnen könnte. ${ }^{1}$

1 Zur Klärung der Begriffe und Termini auf dem Gebiet der Terminologie vgl. besonders Gipper 1964; 1969; Filipec 1969; Renz 1971.

Anmerkung: Erweiterte Fassung eines Referats, das am 9. Juni 1972 vor der Kommission für wissenschaftlich begründete Sprachpflege in Mannheim gehalten wurde. - Ich danke Herrn Dr. F. Strasser von der Psychiatrischen Universitätsklinik in Freiburg für eine freundliche Durchsicht des Manuskripts und für klärende Hinweise.

Ә Open Access. @ Uwe Pörksen, publiziert von De Gruyter. (๔) By-NC-ND Dieses Werk ist lizenziert unter der Creative Commons Attribution-NonCommercial-NoDerivatives 4.0 Lizenz. 
Um in einer Fachwissenschaft z.B. den Begriff ,Widerstand' $z u$ bezeichnen, lässt sich eine Reihe von Möglichkeiten denken:

1. Abkürzungssymbole, Eigennamen und Abkürzungswörter ( $\Omega, \mathrm{Ohm}, W$, WID).

2. Neue Lehnwörter, die aus dem lexikalischen Material der klassischen Gelehrtensprachen oder einer lebenden Fremdsprache entnommen oder geprägt

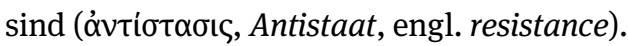

3. Teilweise oder ganz eingebürgerte Lehnwörter in spezialsprachlicher Verwendung (Resistenz, Opposition).

4. Neugebildete Komposita oder feste Lexemsequenzen (Aufhaltekraft, abwehrende Tendenz).

5. Metaphern (Sperre).

6. Gemeinsprachliche Wörter in spezialsprachlicher Verwendung (Widerstand).

7. Vermeidung eines speziellen Terminus durch Synonymik, Paraphrasen und ausgeführte Beschreibungen.

Je weiter wir in dieser Skala nach oben gehen, um so ,künstlicher‘ und weniger allgemeinverständlich sind die Ausdrücke, um so weniger ist ihr Verständnis beeinflussbar durch eine bereits assoziierte Bedeutung und durch die Umgebung eines benachbarten Laut- und Bedeutungsfeldes. Die Skala bezeichnet eine graduelle Entfernung der Termini von der Gemeinsprache in Richtung auf eine Spezial- und Kunstsprache und eine graduell zunehmende Terminologisierbarkeit.

In den zuerst genannten Beispielen, $\Omega, O h m$ und $W$, ist der Ausdruck nahezu beliebig, ein Etikett, das frei ist für die Zuordnung vereinbarter Inhalte und durch eine Definition eingeführt und erlernt werden muss. Je mehr man in der Skala nach unten geht, um so mehr sind die neuen wissenschaftlich intendierten Inhalte erschlossen mit Hilfe eines bekannten Inventars von Ausdrücken und ihnen zugeordneten Vorstellungen, um so mehr sind sie für den Teilhaber der Gemeinsprache motiviert und daher durchsichtig. ${ }^{2}$ Die Ausdrücke der Reihe

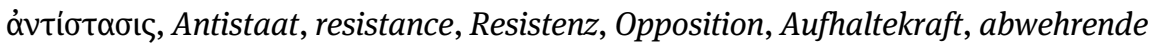
Tendenz, Sperre, Widerstand definieren nicht, enthalten aber einen Hinweis auf den gemeinten Inhalt. In dem unter 7. genannten Beispiel der Umschreibung begrifflicher Inhalte ist die Differenz zur Gemeinsprache aufgehoben.

Allerdings ist $\mathrm{zu}$ sagen, dass jeder gemeinsprachliche Ausdruck in einer Nominal-Definition ohne Rücksicht auf den ihm in der Gemeinsprache zugeord-

2 Über ,durchsichtige ' Wörter als einen zunächst formal bestimmten Typus innerhalb der Wortbildung vgl. Gauger 1970: 113 ff.; Gauger 1971. 
neten Inhalt terminologisiert werden kann. Neben dieser jederzeit möglichen arbiträren Verwendung der Ausdrücke ist aber die Ausnutzung eines gemeinsprachlichen Bedeutungsaspekts ein sehr verbreiteter Typus in der wissenschaftlichen Terminologie (Renz 1971: 311).

Der wissenschaftliche Terminus bewegt sich zwischen den Polen des spezialsprachlichen und arbiträren, nur benennenden, diakritischen, etiketthaften Ausdrucks auf der einen Seite und des gemeinsprachlichen und motivierten, teildefinierenden, klassifizierenden, durchsichtigen und selbsterklärenden Ausdrucks auf der anderen Seite. Die wissenschaftliche Darstellung nähert sich dementsprechend mehr dem spezialsprachlichen Typus und der Verabredungssprache oder der Gemeinsprache.

In einem Lexikon der Physik kommt sie z.B. dem Typus der Verabredungssprache nahe. ${ }^{3}$

In der klassischen Psychopathologie, der Nachbarwissenschaft der Psychoanalyse, sind die Termini üblicherweise dem Lateinischen und Griechischen entnommen (Laplanche \& Pontalis 1972: 8). Bleuler verwendet sie in seinem Lehrbuch der Psychiatrie (1916) in der Weise eines als bekannt vorausgesetzten Vokabulars: „Im chronischen Alkoholismus wird das Gedächtnis ungenau; katathyme Gedächtnisillusionen, deren Inhalt sich scheinbar von der Wirklichkeit weit entfernt, sind nicht selten. In der Dementia alcoholico senilis und im Korsakow kommt die organische Gedächtnisstörung hinzu. Nach dem Delirium tremens ist die Erinnerung eine unvollständige und höchst ungenaue [...]“(Bleuler 1916: 136).

Die von Bleuler gewählte Darstellungstechnik kommt derjenigen nahe, die Freud in Vorüberlegungen zu einem elementaren Abriß der Psychoanalyse (1938) die ,dogmatische“ nennt. In ihr tritt dem Leser der Gegenstand als ,anscheinend in sich geschlossen, fertig“ entgegen, „sie stellt ihre Ergebnisse voran, verlangt Aufmerksamkeit und Glauben für ihre Voraussetzungen, gibt wenig Auskünfte zu deren Begründung“. Ihr stellt er die ,genetische‘ Methode gegenüber, die keine Voraussetzungen macht, sondern von dem allgemein Bekannten ausgeht und dessen Bezirk schrittweise um bisher vernachlässigte oder neue Tatsachen erweitert, aus denen sich für den Leser die Notwendigkeit ergibt, „über sein bisheriges Urteil hinauszugehen, nach neuen Gesichtspunkten zu suchen und neue Annah-

3 Gerlach 1967: 72: „Widerstand heißt die Größe, welche sich für einen Teil eines geschlossenen Stromkreises ergibt, wenn der an ihm liegende Spannungsabfall durch die Stromstärke dividiert wird: V Volt/I Ampere $=\Omega \mathrm{Ohm}$ (Ohmsches Gesetz). Er ist für einen einheitlichen Körper proportional der Länge l, umgekehrt proportional dem Querschnitt q; die Proportionalitätsgröße ist material- und temperaturabhängig; sie heißt der spezifis che Widerstand, wenn $\mathrm{l}=1 \mathrm{~cm}, \mathrm{q}=$ $1 \mathrm{~cm}^{2}$ und die Temperatur $0^{\circ} \mathrm{C}$ (Einheit $\Omega \mathrm{cm}$ ); der reziproke Wert heißt spezifische Leitfähigkeit (Einheit Siemens S/cm).“ 
men zur Erklärung anzuhören. Auf diese Art beteiligt man den Anderen an dem Aufbau einer neuen Theorie des Gegenstandes [...]“ (Freud 1940ff., XVII: 141f.; vgl. Schönau 1968: 35ff.).

Die beiden unterschiedlichen Methoden des deduktiven Lehrvortrags oder der den Leser an der Entwicklung eines neuen Ergebnisses beteiligenden genetischen Darstellung betreffen nicht nur die Textkonstitutionen im Großen, sondern schlagen sich $\mathrm{u}$. U. auch in einer unterschiedlichen Handhabung der Terminologie nieder. Dieser Zusammenhang besteht nicht notwendig, ist aber häufig zu beobachten.

Von einem gegenwärtigen wissenschaftlichen Text würde man eine gewisse Exklusivität erwarten, spezialsprachliche Termini und terminologisierte Gemeinsprache in auffallender Häufigkeit, und eine Darstellungsform, welche mit den Termini - evtl. nach einer einführenden Definition - als autonomen, dem Leser bekannten Vokabeln operiert. Wie verhält sich der Urheber der Psychoanalyse in diesem Punkt?

Freud ist nicht nur durch seine Entdeckungen, sondern auch durch seine schriftstellerischen Qualitäten bekannt. Er schreibt zumeist keine die Kenntnis einer bestimmten Terminologie voraussetzende spezialisierte ,Kunstsprache', sondern eine allgemeinverständliche Bildungssprache, in der er sich der Gebrauchsausdrücke der ,natürlichen Sprache‘ bedient, die Fachtermini erläuternd einführt, sie paraphrasiert und im Zusammenhang der Darstellung durchsichtig werden lässt.

In einem Vortrag von 1919 - also etwa zwanzig Jahre nach dem Bekanntwerden seiner Grundgedanken - äußert er vor Anhängern und „Kollegen“:

\footnotetext{
Wir haben als unsere ärztliche Aufgabe formuliert, den neurotisch Kranken zur Kenntnis der in ihm bestehenden unbewußten, verdrängten Regungen zu bringen und zu diesem Zweck die Widerstände aufzudecken, die sich in ihm gegen solche Erweiterungen seines Wissens von der eigenen Person her sträuben. (Freud 1940ff., XII: 183f.)
}

Dieser allgemeinverständliche und durchsichtige Satz enthält außer dem Wort neurotisch drei Termini technici: ,das Unbewusste‘, Verdrängung', ,Widerstand‘. Durch die Wendungen zur Kenntnis bringen, Erweiterungen des Wissens wird außerdem der Terminus ,Bewusstsein' umschrieben. Die Termini sind aufgrund ihrer usuellen lexikalischen Bedeutung bereits halbwegs verständlich, und sie werden weiter verdeutlicht durch den okkasionellen, aktuellen Zusammenhang, in dem Freud sie verwendet.

Der Hörer wird über das Psychische räumlich orientiert und erhält dabei optische Anhaltspunkte. - Die unbewussten, verdrängten Regungen erhalten das weitere Attribut in ihm bestehend, die Widerstände sträuben sich in ihm. Die Oppositionsbegriffe verdrängt und Erweiterung bestätigen einander in ihrem po- 
tentiellen bildlichen Vorstellungsgehalt, ebenso die den Widerstand ,vergegenständlichenden' Ausdrücke aufdecken und sträuben. Die Vorstellungen evozieren und stützen einander wechselseitig und sind konsequent eingebaut in eine verräumlichte Gesamtvorstellung von der Psyche als einem Ort des verdrängten Unbewussten, des zu erweiternden Wissens und eines zwischen beiden liegenden, von der Person des Kranken gespeisten Widerstandes.

Dabei haben die Wörter Unbewusstes, Verdrängung, Widerstand die Qualität von Begriffen. Freud verwendet sie in einem spezialisierten Sinn, sie erfahren, mit Hermann Paul (1970: 87) gesprochen, der sich hier an die in der Logiktradition übliche Unterscheidung zwischen Extension (Umfang) und Intension (Inhalt) der Begriffe anschließt, eine „Spezialisierung der Bedeutung durch Verengung des Umfangs und Bereicherung des Inhalts“. Das Wort Widerstand bezeichnet allgemein ,ein Verhalten von lebendigen Wesen oder von leblosem Material, das eine Bewegung, einen Strom, eine arbeitende Kraft hemmt oder aufhält‘. Diese weitgespannte, abstrakte Bedeutung kann sich nun, je nach Gelegenheit, konkretisieren und präzisieren (vgl. Weinrich 1966: 30), z.B. als Widerstand einer Stadt gegen eine Belagerung, eines Körpers gegen eine Krankheit, eines Gebäudes gegen den Zerfall, einer Gruppe von Schülern gegen ihren Lehrer. Um das Wesen des Terminus $\mathrm{zu}$ beschreiben, halte ich die Unterscheidungen Hermann Pauls zwischen ,usueller' und ,okkasioneller' Bedeutung ${ }^{4}$ für am besten geeignet. Der Freud'sche Begriff ,Widerstand“ bezieht sich auf einen Typus von ,Gelegenheiten“, und zwar wird er ständig für die Beziehung des Patienten zu dem Analytiker gebraucht. Freud (1940ff., II/III: 521) definiert: „Was immer die Fortsetzung der Arbeit stört, ist ein Widerstand“. Dadurch ist sein Bedeutungsumfang eingeschränkt und normiert. In diesem festliegenden semantischen Geltungsbereich (Ullmann 1967: 58) ist er gleichzeitig um eine vorher gar nicht bekannte Dimension erweitert, eben um den von Freud bei der psychoanalytischen Arbeit entdeckten Widerstand gegen unbewusste Regungen. Das Vokabular der Psychoanalyse erweitert die Definition: „Im Verlauf der psychoanalytischen Behandlung nennt man all jenes ,Widerstand', was in den Handlungen und Worten des Analysierten sich dem Zugang zu seinem Unbewußten entgegenstellt“ (Laplanche \& Pontalis 1972: 622). Der Freud'sche Terminus bezieht sich also in seinem Umfang auf einen bestimmten, aber typischen Realitätsausschnitt und er bezeichnet inhaltlich ein „systemhaftes Merkmal“ (Filipec 1969: 410) bzw. einen Merkmalskomplex, der durch den engeren Kontext der angrenzenden Begriffe (z.B. das Unbewusste) und den weiteren des Freud'schen Lehrgebäudes definiert ist.

4 Paul 1970: 75f. Vgl. Schmidt 1963 ,lexikalische‘ und ,aktuelle‘ Bedeutung und Weinrich 1966 ,Bedeutung، und ,Meinung‘. 
Freud verwendet Termini, aber er schreibt wenig terminologisch. Er gebraucht die Termini nicht in der Art spezialsprachlicher, etikettierender Namen, deren Bedeutung als bekannt vorausgesetzt wird, sondern lässt ihre Bedeutung im Darstellungszusammenhang erschließbar werden. Darin liegt m. E. die besondere Qualität seines wissenschaftlichen Sprachstils. Das Verfahren wird durch den Typus seiner Termini und die Art ihrer Verwendung ermöglicht. Er bedient sich überwiegend allgemein bekannter, oft bildlicher Ausdrücke der Gemeinsprache und aktualisiert die spezifische Bedeutung, die sie in seinem Begriffssystem haben, indem er den diese Bedeutung definierenden Kontext mitliefert: der okkasionelle Gebrauch ist geregelt und die Begriffsdefinitionen - sozusagen die Gebrauchsregeln des künstlich geschaffenen Vokabulars der Psychoanalyse werden im sprachlichen Kontext meist so weit wiederholt, dass die Meinung der Termini verständlich ist; in dem oben zitierten Satz wird das gesamte den Begriff ,Widerstand‘ definierende Begriffssystem zitiert.

Die Begriffsdefinitionen sind allerdings nicht immer genau und nicht jeweils an einen bestimmten Ausdruck gebunden. Charakteristisch für Freuds Darstellungsstil, was hier nur angedeutet werden kann, sind z.B. Variation und Umschreibung. Er variiert etwa zwischen ,Abwehr' und ,Widerstand' (Freud 1940ff, I: 280, 285), ,Libido‘ und ,Tätigkeit der Lebenstriebe‘ (Freud 1940ff., XIII: 54), oder erläutert den Begriff der ,Traumzensur‘: „Der Zweifel an der richtigen Wiedergabe des Traums oder einzelner Traumdaten ist wieder nur ein Abkömmling der Traumzensur, des Widerstands gegen das Durchdringen der Traumgedanken zum Bewußtsein“ (Freud 1940ff., II/III: 520). Der einzelne Terminus kann durch Synonyma ersetzt werden, ,Dingvorstellung d durch ,Sachvorstellung‘, ,Aufarbeitung“ durch ,Ausarbeitung', ,Bearbeitung‘, ,Verarbeitung‘, ,Bewußtheit‘ durch ,Bewußtsein', ,Überdeterminierung' durch ,mehrfache Determinierung‘. Der fließende Übergang zwischen einer terminologischen Fachsprache und einer unspezifischen Gemeinsprache lässt sich schon an diesem Terminologiegebrauch ablesen. Lagache schreibt im Vorwort des Vokabulars der Psychoanalyse: „Wenn Freud sich als Schriftsteller auch erfindungsreich zeigte, so war er doch um die Perfektion seines Vokabulars wenig besorgt. [...] Ein Wort hat mehrere Bedeutungen, und es kommen semantische Überschneidungen vor. Hingegen werden nicht unbedingt differente Ideen oft in mehreren Worten ausgedrückt“ (Laplanche \& Pontalis 1972: 8).

Die Nähe zum gemeinsprachlichen und orientierenden Terminologietypus zeigt sich auch in der formalen Struktur zahlreicher Termini. Freud verwendet zu einem Fünftel feste Lexemsequenzen, welche die Qualität eines Begriffs haben, und zwar hauptsächlich Verbindungen von Substantiven mit Adjektivattributen (52), z.B. ,kathartische Methode‘, ,phallische Stufe', ,mütterliches Betreuen', an zweiter Stelle Verbindungen mit Genitivattributen (10), z.B. ,Abkömmling des 
Unbewußten', ,Abziehen der Besetzung', und schließlich solche mit Präpositionalattributen (5), z. B. ,Flucht in die Krankheit‘, ,Identifizierung mit dem Angreifer‘ Sie erfüllen den Typus der motivierten, durchsichtigen Termini in reiner Form.

Von den 331 Termini, die in dem Vokabular der Psychoanalyse erläutert werden, sind 110, also genau ein Drittel, nur aus Erbwörtern des Deutschen gebildete Ausdrücke, eine auffallend hohe Zahl. Es handelt sich um einfache Wörter des Gemeinwortschatzes, wie z.B. ,Lust', ,Abwehr', ,Begierde‘, ,Trieb‘, ,Verneinung', ,Wunsch', ,Zärtlichkeit', um neue Wortbildungen wie ,Fehlleistung‘, ,Deckerinnerung', ,Gegenbesetzung', ,Bemächtigungstrieb‘, ,Traumarbeit‘, ,Trauerarbeit' und um die bereits zitierten Lexemsequenzen.

Neben diesen sozusagen muttersprachlichen Termini gibt es als zweiten Typ die Verbindung von deutschsprachigen Elementen mit mehr oder weniger eingebürgerten Lehnwörtern aus dem Griechischen oder Lateinischen oder mit aus diesen Sprachen gebildeten speziellen Fachtermini: ,Böses Objekt', ,seelischer Apparat', ,Lustprinzip“, ,Primärvorgang', ,Harnerotik', ,Destruktionstrieb', ,Abwehrmechanismen', ,Ichideal', ,Wahrnehmungsidentität‘, ,Penisneid‘, ,latenter Traumgedanke‘, .manifester Inhalt' sind Beispiele für das Erstere. Daneben stehen dann die Verbindungen mit spezialsprachlichem lateinisch-griechischem Wortmaterial: ,Klebrigkeit der Libido', ,Ichlibido‘, ,Eltern-Imago‘, ,analsadistische Stufe', ,orale Stufe', ,oralsadistische Stufe‘, ,Abwehr-Neuropsychose‘. Die eigentümliche Zwischenstellung der Sprache Freuds zwischen wissenschaftlicher Kunstsprache und ,natürlicher' Bildungssprache wird an diesen Beispielen besonders deutlich. Es sind annähernd hundert, also etwas weniger als ein Drittel der Termini des Vokabulars der Psychoanalyse.

Etwas mehr als ein Drittel, genau 122 Termini dieses Wörterbuchs sind Ausdrücke, die ganz auf fremdsprachliches Material, vor allem auf das Lateinische und Griechische, zurückgehen. Ein guter Teil dieser von Freud benutzten Wörter ist um 1900 gemeinsprachlich oder zumindest bildungssprachlich verbreitet: $A b$ stinenz, Zone, Apparat, Zensur, Analyse, Fixierung, Instinkt, Objekt, Material, Kloake, Theorie, Ödipus, Komplex, psychisch, Konflikt, spezifisch, Aktion, Phantasie - diese Wörter unterscheiden sich nicht oder nur kaum von den übrigen terminologischen Ausdrücken Freuds und werden von ihm in der gleichen Weise verwendet. Ihre terminologisch geregelte Bedeutung wird bei Gelegenheit ihres Gebrauchs aktualisiert wie die der übrigen Termini: „Wir sagen, auch die ausgelassenen, durch ein Gemurmel verhüllten Traumreden sind einer Zensur zum Opfer gebracht worden. Wir sprechen direkt von einer Traumzensur, der ein Stück Anteil an der Traumentstellung zuzuschreiben ist.“ (Freud 1940ff., XI: 139f.)

Die Abweichung Freuds von dem terminologischen Typus der heutigen Wissenschaftssprache ist auffallend - wie weit auch von der Wissenschaftssprache 
der Jahrhundertwende, wäre zu prüfen. Bei dem gesamten Lehngut des Vokabulars handelt es sich überwiegend um mehr oder weniger eingebürgerte Lehnwörter, die in dem 1891 erschienenen, seinem Programm (Sanders 1891, I: VIf.) nach nicht fachsprachlich, aber de facto zumindest bildungssprachlich orientierten Fremdwörterbuch von Sanders angeführt sind. Der kleinere Teil neuer, aus fremdsprachlichem Material geprägter oder entnommener Lehnwörter stammt nur etwa zur Hälfte von Freud (20), der Rest (25) von seinen Kollegen und Schülern, von Breuer, Abraham, Jung, Low, Jones, Spitz. Die Terminologie der Psychoanalyse ist nach Freud fast ausschließlich in Richtung auf den lehenssprachlichen terminologischen Typus erweitert worden. ,Anaklitisch', ,Aphanisis', ,Internalisierung', ,Urethralerotik‘ (statt Freuds ,Harnerotik') sind Beispiele.

Von Polenz (1967a) hat mit Recht betont, dass die eingebürgerten Lehnwörter, abgesehen von den nur in Sektoren der Fachwissenschaft bekannten ,difficult words', in der Gemeinsprache nicht anders verwendet werden als das übrige allgemein bekannte Wortgut, und nicht als ,Fremdwörter deklariert werden könnten. Dem entspricht auch die Verwendungsweise Freuds. Man kann sich aber fragen, ob dieser Worttyp im Kontext einer wissenschaftlichen Darstellung nicht trotzdem eine Sonderstellung behauptet.

In der wissenschaftlichen Fachsprache ist auch nach der Ablösung aus der lateinischen Gelehrtensprache der aus griechisch-lateinischem Wortmaterial geprägte Fachterminus üblich - auf das eingebürgerte Lehnwort aus diesen Sprachen richtet sich deshalb, aufgrund paradigmatischer Analogie, die gleiche Vorerwartung, dass es sich um einen enger gebrauchten Fachausdruck handele.

Es ist gerade den wissenschaftlichen Sprachteilhabern oft in der Bedeutung, die es in seiner Herkunftssprache hat, durchsichtig und wird deshalb $u$. U. genauso angewendet wie die aus dem fremdsprachlichen Vokabular neu gebildeten Termini.

Der ,Fremdwörterstil` ist ein Kennzeichen der wissenschaftlichen Zunftsprache, er hat eine gruppenabgrenzende und -stabilisierende Funktion. Auch aus Gründen der Textsortentradition wird man veranlasst zum seiner Herkunft nach fremdsprachlichen Terminus. In der Umgebung der entlehnenden Sprache steht das Fremdwort in der Regel in einem schwächeren Assoziationsgefüge, in der Wortbildung ist sein Etymon weniger produktiv, es ist im Laut- und Bedeutungsfeld der Sprache isolierter und deshalb u. U. weniger störenden Assoziationen ausgesetzt, also als Terminus geeigneter.

Vor allem ist es leicht übersetzbar, weil das Wort in den anderen europäischen Sprachen oft in der entsprechenden Bedeutung vorhanden ist oder als assimilierter Typus übernommen werden kann. Lagache bemerkt in der Einleitung zum Vokabular der Psychoanalyse: Freud „,nahm seine Worte und Formulierungen vorwiegend aus dem Deutschen und schöpfte aus den Möglichkeiten, die 
seine eigene Sprache ihm bot. So kommt es, daß eine genaue Übersetzung in andere Sprachen schwierig ist und die analytische Sprache oft einen ungewöhnlichen Eindruck vermittelt, was bei Freuds Sprache nicht der Fall ist [...]“ (Laplanche \& Pontalis 1972: 8). Es ist gelegentlich schwierig, für die deutschen Ausdrücke das Äquivalent in der Übersetzungssprache $\mathrm{zu}$ finden und eine einheitliche Terminologie durchzusetzen.

Der Begriff ,Anlehnung‘ wird z.B. französisch uneinheitlich wiedergegeben, u.a. als ,étayage‘. Der Begriff ,Anlehnungstypus der Objektwahl` wird allgemein übersetzt mit ,type anaclitique de choix d'objet‘, die verbale Form ,sich (an etwas) anlehnen' u.a. mit ,être attaché à', être basé sur‘, ,prendre appui sur‘. Die Folge dieser Uneinheitlichkeit ist, „daß der Begriff ,Anlehnung‘ Freuds Lesern nicht deutlich genug werden konnte“ (Laplanche \& Pontalis 1972: 61). Die LehnwortTermini Freuds werden demgegenüber im Englischen, Französischen, Italienischen, Portugiesischen, Spanischen prinzipiell mit dem gleichen Wortmaterial wiedergegeben. Ein beliebiges Beispiel: dt. ,psychische Repräsentanz‘, engl. ,psychical representative‘, frz. ,représentant psychique‘, ital. ,rappresentanza psichica‘ oder ,rappresentante psichico‘, port. ,representante psíquico‘, span. ,representante psíquico'. 5

Ein Charakteristikum in dem Werk Freuds sind Wendungen, in denen ein neuer Fachausdruck eingeführt und ausdrücklich terminologisiert wird. Die Definitionen können die Festlegung von Sachverhalten implizieren und die Form von Gesetzesdefinitionen annehmen. „Man nennt diese Fähigkeit, das ursprünglich sexuelle Ziel gegen ein anderes, nicht mehr sexuelles, aber psychisch mit ihm verwandtes, zu vertauschen, die Fähigkeit zur Sublimierung“ (Freud 1940ff., VII: 150). Schönau (1968: 36f.) weist darauf hin, dass bei Freud die ,genetische“ Darstellungstechnik vor der ,dogmatischen“ überwiegt. Er nennt als Beispiel für die letztere die streng systematischen Drei Abhandlungen zur Sexualtheorie (1905) und die erste Fassung des Abriß der Psychoanalyse (1938), andere wären das 7. Kapitel der Traumdeutung (1900) und Partien von Das Ich und das Es (1923). In diesen deduktiv von Resultaten ausgehenden Abhandlungen nähert sich Freud gleichzeitig häufiger einer mit einem vordefinierten Vokabular operierenden, terminologischen Schreibweise.

Im 7. Kapitel der Traumdeutung gibt er eine versuchsweise Beschreibung des ,psychischen Apparats‘. Das Bauprinzip eines aus Linsensystemen zusammengesetzten Apparats (Fernrohr, Mikroskop) ist das allgemeine Modell für diese „Annäherung an etwas Unbekanntes“. Freud verwendet hier die Abkürzungssymbole

5 Die Übersetzungen von Freuds Termini würden eine eigene Untersuchung lohnen; das Problem sollte hier nur angedeutet werden. Vgl. z.B. den Artikel „Phantasie“. 
System, Vbw, Ubw, $W(W=B w), E r$ und arbeitet mit Skizzen (Fig. 1, 2, 3). Die Diktion ist an die eines Lehrbuchs der Physik angelehnt (Freud 1940ff., II/III: 542ff.; vgl. XIII: 252f.). Nicht nur der physikalische Apparat, auch die Form seiner Beschreibung hat hier den Charakter der Analogie. Man könnte von einer Textsortenübertragung ${ }^{6}$ sprechen.

Freud nannte die Drei Abhandlungen zur Sexualtheorie (1905) das ,an die Biologie angrenzende Stück der Lehre“ (Freud 1940ff., V: 31, 30). Der Teil I behandelt ,Die sexuellen Abirrungen‘. Er beginnt: „Die Tatsache geschlechtlicher Bedürfnisse bei Mensch und Tier drückt man in der Biologie durch die Annahme eines ,Geschlechtstriebes‘ aus“ (Freud 1940ff., V: 33). Freud definiert dann die Termini ,Sexualobjekt‘ und ,Sexualziel‘ und behandelt unter 1) ,Abweichungen in Bezug auf das Sexualobjekt‘. Er äußert sich über das Phänomen der Homosexualität. „Man heißt solche Personen Konträrsexuale oder besser Invertierte, die Tatsache die der Inversion.“ Es folgt: „A) Die Inversion. Die betreffenden Personen verhalten sich nach verschiedenen Richtungen ganz verschieden. a) Sie sind absolut invertiert, das heißt ihr Sexualobjekt kann nur gleichgeschlechtlich sein [...] b) Sie sind amphigen invertiert, das heißt [...] c) Sie sind okkasionell invertiert, das heißt [...] Die Invertierten zeigen ferner ein mannigfaltiges Verhalten [...] Weitere Variationen betreffen die zeitlichen Verhältnisse [...]“ (Freud 1940ff., V: 34f.). Eine strikte Systematik gliedert das „Material“. Das Verfahren ist deduktiv. Die allgemeinen Tatsachen werden vorangestellt und expliziert, die Termini eingeführt und dann mit ihnen gearbeitet. Die Tatsachen des menschlichen Sexualverhaltens werden vollständig beschrieben, auch wenn sie allgemein bekannt sind (vgl. Freud 1940ff., V: 47ff.). Der Autor verhält sich emotionslos neutral und lehnt jede „Parteinahme“ ab (Freud 1940ff., V: 51). Er verhält sich wie ein biologischer Systematiker, der eine neue noch unbekannte Art beschreibt; man möchte an Linnés ,vollständiges Pflanzensystem“ erinnern: „Die Vermehrung erfolgt bei den Pflanzen auf zwei Arten: 1. Durch Samenbildung, 2. durch Schößlinge, Pfropfreiser, Teilung und dergleichen.“ (Dannemann 1902: 128f.) Damit ist keine Beeinflussung durch Linné gemeint, eher könnte der von Freud bewunderte Darwin als stilistisches Vorbild auf ihn gewirkt haben.

Die Form der Systematik und Einführung der Termini, die Neutralität des Beobachters, die idiomatischen Wendungen und Vokabeln, etwa der für die Biologie typische Begriff der ,Annahme‘, der biologische ,Geschlechtstrieb‘, der Begriff des ,Objekts‘, der ,Variationen“ und ,Reihen“, das ,sich verhalten“, ,ein Verhalten zeigen', ,beobachtet werden', bauen einen vorwiegend biologischen

6 Zum Begriff, Textsorte‘ vgl. Steger 1970: 17, 55; 1971: 18ff. 
Textsortentypus auf, der hier, auf den ersten Seiten der Abhandlung, in extremer Weise auf das Gebiet des menschlichen Verhaltens projiziert ist.

Innerhalb der Terminologie verdienen die der Naturwissenschaft entlehnten Analogien besonderes Interesse. Ein erheblicher Teil der Termini lässt sich den drei grundlegenden Vorstellungskategorien der Psychoanalyse zuordnen: der ,dynamischen', welche sich die psychischen Phänomene als Ergebnis eines Konflikts widerstreitender Seelenkräfte vorstellt, der ,ökonomischen', die bei den psychischen Vorgängen ausgeht von messbaren oder in ihrer Quantität jedenfalls abschätzbaren Energien, die im psychischen Apparat umlaufen und sich verteilen, erhöht oder verringert werden können, und der ,topischen', in der die psychischen Systeme des ,Unbewußten“, ,Vorbewußten‘, ,Bewußten“ als räumlich aufeinander bezogene Orte vorgestellt werden. Die folgenden Ausdrücke lassen sich in einem verräumlichten Modell lokalisieren, in dem quantitativ abschätzbare, dynamische Energiebewegungen vor sich gehen: ,verdrängte‘, ,unbewußte‘ Inhalte, die erst nach Überwindung eines ,Widerstandes` dem ,Bewußtsein“ zugänglich werden, ,psychische Konflikte‘, die ,Abfuhr eingeklemmter Affekte‘, das ,Abreagieren', die ,Verschiebung“ energetischer Ladung, ,Erregungssummen‘, die ,Besetzung“ von Vorstellungen mit affektiver Energie, die Tendenz des ,psychischen Apparates‘, die in ihm zirkulierenden Energien möglichst niedrig zu halten, die ,Verarbeitung‘ der Erregungen, das Spiel energetischer ,Besetzungen“, ,Gegenbesetzungen‘, die ,Umwandlung‘ von freier in gebundene Energie, die ,Schichtung‘ des Unbewußten, die räumlich aufeinander bezogenen psychischen Systeme des ,Unbewußten“, ,Vorbewußten“ und ,Bewußten“ und die ,Zensur' an den Übergangsstellen zwischen den Systemen, die eine Kontrollfunktion wahrnimmt. Diese Ausdrücke sind überwiegend der Physik entlehnte Gleichnisse. Freuds Terminologie schließt sich zu einem Teil an naturwissenschaftliche, speziell physikalische Vorstellungsweisen an. Die Begriffe aus der Naturwissenschaft gehen dabei auf recht allgemeine, vermutlich um die Jahrhundertwende zur Allgemeinbildung gehörende naturwissenschaftliche Vorstellungen zurück - insofern unterscheiden sie sich wohl nicht von dem allgemeinen Gebrauch von Metaphern, deren Funktion es ist, unbekanntes Gelände mit Hilfe bekannter Vorstellungen zu erschließen.

Freud benutzt seine Ausdrücke nicht nur in ihrem kognitiven Gehalt, sondern berücksichtigt - nach Werken verschieden - die gemeinsprachlich mit ihnen assoziierten Gefühlselemente. Es wäre eine eigene Untersuchung, in welchem Umfang ein solches emotives Konnotat ${ }^{7}$ der Termini in den verschiedenen $\mathrm{Zu}$ sammenhängen und Werken aktualisiert wird. Die Anwesenheit solcher Elemente

7 Literatur bei Ullmann 1967: 90ff.; Hörmann 1970: passim. 
ergibt sich auch aus dem Bildgehalt der Sprache Freuds. Anschauliche Sprache und emotiver Gehalt sind enger verbunden als kognitive Sprache und emotiver Gehalt, das zeigt sich schon an der Neigung der Polemik zur anschaulichen Sprache.

In den Vorlesungen (1917) wird z.B. der Terminus ,Zensur‘ in einem ausführlichen Vergleich mit der - gefühlsbelasteten - Zeitungszensur eingeführt (Freud 1940ff., XI: 139). Wenn Freud das psychologische Vokabular der Umgangssprache, etwa ,Zärtlichkeit‘, ,Lust‘, ,Phantasie‘, ,Wunsch“, terminologisch verwendet, so erweitert er es nach der kognitiven Seite, verzichtet aber nicht auf Aspekte des Ausdrucks einer gefühlsmäßigen Einstellung zu dem Inhalt oder des Appells an Gefühle des Lesers. Er entwickelt den Terminus ,Wunscherfüllung' nahtlos aus der persönlich getönten Gemeinsprache „Auch ist mir unterdes der ,Sinn“ des Traumes aufgegangen. Ich habe eine Absicht gemerkt, welche durch den Traum verwirklicht wird und die das Motiv des Träumens gewesen sein muß. Der Traum erfüllt einige Wünsche, welche [...] in mir rege gemacht worden sind [...]. Der Traum stellt einen gewissen Sachverhalt so dar, wie ich ihn wünschen möchte, sein Inhalt ist also eine Wunscherfüllung, sein Motiv ein Wunsch“ (Freud 1940ff., II/III: 123). Das ,Es` wird mit den Worten eines anderen Autors eingeführt, „daß wir ,gelebt“ werden von unbekannten unbeherrschbaren Mächten“, und in einer Anmerkung wird an Nietzsches Gebrauch des Ausdrucks „für das Unpersönliche und sozusagen Naturnotwendige in unserem Wesen“ erinnert (Freud 1940ff., XIII: 251). In den Termini vom Typ ,Lustprinzip“, ,somatisches Entgegenkommen', ,Aggressionstrieb“, ,Identifizierung mit dem Angreifer‘ verbindet er sehr allgemeine muttersprachliche Wörter mit Lehnwörtern, bei denen im Kontext wissenschaftlicher Darstellung eine Vorerwartung in Richtung auf Terminologie besteht, bedient sich also des Mittels der Kontrastierung von Wörtern aus verschiedenen Sphären mit unterschiedlichen emotiven Konnotaten. Man könnte von einer Form der Metapher sprechen. ${ }^{8}$ Ullmann (1967: 94) erläutert die Wirkung: „Die gefühlsmäßige Wirkung dieser Elemente beruht auf ihrem Evokationsvermögen: Sie erinnern uns an die Umgebung bzw. an die Stilschicht, in die sie normalerweise hineingehören. Bally spricht hier von ,effets par évocation d'un milieu“".

Muschg (1958: 317) spricht anlässlich der Zusammensetzungen mit dem Wort ,Traum“ von „Wortzauber als Begleiter und Diener einer Erkenntnis“, von dem Sinn Freuds für die Lautschicht und die Klangreize der Sprache (Muschg 1958:

8 Weinrich in: Bochumer Diskussion 1968: 100: „Ein Wort in einem Text setzt eine bestimmte Kontexterwartung, die von dem tatsächlichen Kontext enttäuscht werden kann. Die Metapher ist definierbar als ein Wort in einem konterdeterminierenden Kontext.“ 
305f.), seinem „Hang, schlagende Worte zu formen“ (Muschg 1958: 317). Er beobachtet (Muschg 1958: 305) „Schönheit und Schlagkraft der Formulierung, rhythmische und klangliche Sicherheit“" in der Titelgebung (Jenseits des Lustprinzips, Das Unbehagen in der Kultur). Die Sprache Freuds steckt voller Ausdrucks- und Appellwerte, der Leser wird nicht nur in einer kognitiven, sondern auch in einer emotiven Schicht angesprochen.

Der allgemeinverständliche und plastische Stil, von dem eingangs die Rede war, erklärt sich also u.a. daraus, dass Freud bei der Wahl seiner Spezialausdrücke auf die Gemeinsprache (Bildungssprache) zurückgreift und auch bei ihrem Gebrauch insofern gemeinsprachlich (bildungssprachlich) bleibt, als er häufig ihre usuelle Bedeutung im konkreten Sachzusammenhang in verständlicher Weise spezialisiert, den sprechenden, oft bildlichen Gehalt der Wörter aktualisiert. Ausgeführte Gleichnisse, Metaphern, Vergleiche mit bekannten Gegenstandsbezirken (Muschg 1958: 325ff.; Schönau 1968: Kap.7), speziell eine zusammenhängende Gruppe von der Physik entlehnten Analogien, machen die Darstellung durchsichtig. Diese Spezialausdrücke sind auf der anderen Seite wissenschaftliche Termini, da der sachliche Kontext, in dem sie auftreten, und die Bedeutung, in der sie gebraucht werden, verbindlich geregelt sind.

Die in dieser Wissenschaftssprache dargestellten Inhalte werden vorstellbar, das heißt wohl, dem Nacherleben der psychischen Vorgänge stärker zugänglich. Freud aktiviert zugleich mit der intellektuellen Einsicht des Lesers eine emotionale Schicht. Sein Darstellungsstil verwissenschaftlicht das Erleben und sichert einer wissenschaftlichen Analyse die Beibehaltung eines Erlebnisanteils, an dem der Leser partizipieren kann.

Freuds Sprache steht dadurch genau auf der Grenze zwischen wissenschaftlichem und schriftstellerischem Sprachstil. Gelegentlich nähert er sich mehr dem wissenschaftlichen Typus, streckenweise, wo Krankengeschichten vorgetragen werden, gerät er ganz auf die schriftstellerische Seite, was er an einer frühen Stelle (1895) mit einer gewissen Beunruhigung ausgesprochen hat.

Ich bin nicht immer Psychotherapeut gewesen, sondern bin bei Lokaldiagnosen und Elektroprognostik erzogen worden wie andere Neuropathologen, und es berührt mich selbst noch eigentümlich, daß die Krankengeschichten, die ich schreibe, wie Novellen zu lesen sind, und daß sie sozusagen des ernsten Gepräges der Wissenschaftlichkeit entbehren. Ich muß mich damit trösten, daß für dieses Ergebnis die Natur des Gegenstandes offenbar eher verantwortlich zu machen ist als meine Vorliebe; Lokaldiagnostik und elektrische Reaktionen kommen bei dem Studium der Hysterie eben nicht zur Geltung, während eine eingehende Darstellung der seelischen Vorgänge, wie man sie vom Dichter zu erhalten gewohnt ist, mir gestattet, bei Anwendung einiger psychologischer Formeln doch eine Art von Einsicht in den Hergang einer Hysterie zu gewinnen. (Freud 1940ff., I: 227) 
In dem Abschnitt sind bewusst kontrastierend Vokabeln aus der objektiv wissenschaftlichen unemotionalen Sphäre (Lokaldiagnose, Elektroprognostik, ...) und solche aus dem persönlich lebensgeschichtlichen gefühlsverbundenen Ausdrucksbereich (Ich bin nicht immer, bin erzogen worden, es berührt mich, wie Novellen zu lesen, tröstet mich, meine Vorliebe, ...) miteinander verbunden. Es ist ein Beispiel für die Verschränkung zweier Textsorten. ${ }^{9}$ Am Schluss charakterisiert Freud mit den Worten „eingehende Darstellung der seelischen Vorgänge“ und „psychologische Formeln“ die von ihm intendierte und später ausgearbeitete Verbindung des schriftstellerischen und wissenschaftlichen Texttyps. Lassen sich für die Wahl dieses Darstellungsstils Gründe angeben?

Freud entschuldigt sich mit der Natur des Gegenstandes, d.h. den Krankengeschichten, dem Hergang einer Hysterie. Die psychologischen Formeln, von denen er spricht, bezeichnet Habermas (1970: 309ff.) einleuchtend als ,allgemeine Interpretationen“. Der Gegenstand des Analytikers sind ihm zufolge die „Bildungsprozesse“ von Individuen, also Historisches. In den ,allgemeinen Interpretationen“ wird das individuelle Historische systematisch verallgemeinert $\mathrm{zu}$ einem das Typische kennzeichnenden Auslegungsschema für neue individuelle Geschichten. Die Geltung dieser Interpretationen ist nun nach Habermas davon abhängig, dass sie von den betroffenen Personen auf sich selbst angewendet werden. Ihre „empirische Triftigkeit“ hängt „allein von der vollzogenen Selbstreflexion und einer anschließenden Kommunikation zwischen dem Forscher und seinem ,Objekt‘ ab“ (Habermas 1970: 318f.). Das hat Konsequenzen für die Sprache:

Der Gebrauch einer terminologisierten Umgangssprache kennzeichnet nicht einen zufälligen Entwicklungsstand der Psychoanalyse. Alle Versuche, der Metapsychologie eine strengere Form zu geben, sind gescheitert, weil die Bedingungen der Anwendung allgemeiner Interpretationen eine Formalisierung der Umgangssprache ausschließen. Die darin verwendeten Terme dienen nämlich der Strukturierung von Erzählungen; an sie wird in der Umgangssprache des Patienten angeknüpft, wenn beide, Arzt und Patient, das analytische Erzählschema zu einer Geschichte komplettieren. (Habermas 1970: 322f.)

Das Gesagte lässt sich auf die Beziehung zwischen dem psychoanalytischen Forscher und seinem Leser ausweiten. Auch der Leser überprüft die Darstellung mit gemeinsprachlich gestalteter Erfahrung - als Analytiker oder in begrenztem Rahmen als Laie - und mit Selbstreflexion. (Freud übertrug den analytischen Begriff des Widerstandes aus der ärztlichen Praxis auf die Oppositionshaltung gegen

\footnotetext{
9 Vgl. die Vorbemerkung der Traumdeutung: „Mit der Mitteilung meiner eigenen Träume aber erwies sich als untrennbar verbunden, daß ich von den Intimitäten meines psychischen Lebens fremden Einblicken mehr eröffnete, als mir lieb sein konnte und als sonst einem Autor, der nicht Poet, sondern Naturforscher ist, zur Aufgabe fällt“ (Freud 1940ff., II/III: VIII).
} 
seine Veröffentlichungen.) Der Aufgabe, systematisch zu verallgemeinern und die gemeinsprachlich sich gestaltende Erfahrung und Selbstreflexion anzusprechen, entspricht der beschriebene Typus von Freuds Wissenschaftssprache.

Freud erwähnt außer der Natur des Gegenstandes Gründe der Textsortentradition, die seine Darstellungsweise nahelegen. Das Gebiet, mit dem er sich beschäftigt, fällt bisher eher in die Observanz der Dichtung als in die der Neuropathologie. Sein Beobachtungsmaterial, z. B. die freie Assoziation der Patienten, der Traum, die Fehlleistungen des Alltagslebens, der Witz, sind bis dahin von der Wissenschaft vernachlässigt oder nicht entdeckt. Freud kann daher auf keine ausgebildete Terminologie zurückgreifen, sondern muss sie in dem von ihm begründeten und sich erweiternden Wissenschaftszweig erst schaffen. Dabei veranlasst ihn vielleicht die Natur des wissenschaftlich nicht ernst genommenen Gegenstandes $\mathrm{zu}$ einer besonders strengen wissenschaftlichen Behandlung, auf der anderen Seite kann er sich nicht an ein Fachpublikum wenden, das sich bereits in einer einschlägigen Zunftsprache bewegt, sondern muss einem gemeinsprachlichen Leser eine an allgemein menschliche Phänomene anknüpfende neue Theorie vermitteln. Das legt auch eine gemeinsprachliche Terminologie nahe. Vielleicht ist auch sein „gebrochenes“ Verhältnis zur Wissenschaft (vgl. Scharfenberg 1969: 54ff.), ein in seiner Biographie und in seiner Konstitution begründeter Affekt gegen die Fachwelt ein wesentlicher Grund für seine wenig „zünftige“ Sprache.

Die Traumdeutung (1900) wendet sich in der Vorbemerkung an die Kollegen von der Psychiatrie, im Vorwort zur zweiten Auflage (1908) spricht Freud von dem Desinteresse der Fachkreise und nennt als Leser die „kleine Schar“ von Anhängern unter den Arztkollegen und die „weiteren Kreise von Gebildeten und Wißbegierigen“. Welcher Art das in den verschiedenen Werken intendierte Publikum ist, in welcher Weise das Bewusstsein eines allgemeinen Interesses seiner Mitteilungen den Darstellungsstil Freuds bestimmte und wie seine Wirkung in der Öffentlichkeit auf seinen Stil zurückwirkte, bedürfte einer eingehenderen Untersuchung.

Eine speziellere Frage wäre, warum Freud seine psychologischen Erkenntnisse in zahlreichen Analogien auseinandersetzte.

Gipper (1969: 70f., 75f.) macht darauf aufmerksam, dass Metaphernreichtum ein Kennzeichen junger Fachsprachen ist. Die Geschichte der Anatomie beginne mit grober Metaphorik und anthropomorphen Vorstellungen. Faraday und Einstein seien zwei illustre Beispiele dafür, wie mit der Hilfe anschaulicher Bilder und Metaphern neue Sehweisen versprachlicht werden könnten. Der Zusammenhang leuchtet ein. Bei der Erschließung eines neuen wissenschaftlichen Geländes knüpft man am einfachsten an Bekanntes an, um eine Vorstellung von dem Unbekannten zu vermitteln.

In der Psychologie erscheint nun das Denken in Analogien als geradezu unvermeidlich, weil man zwar die Resultate psychischer Vorgänge greifen kann, 
aber nicht diese Vorgänge selbst. Freud beschreibt die Entstehung der ,Abwehrneuropsychosen“ (1894): „[...] dies sind Vorgänge, die ohne Bewußtsein geschehen, die man nur supponieren, aber durch keine klinisch-psychologische Analyse erweisen kann. Vielleicht wäre es richtiger zu sagen: Dies sind überhaupt nicht Vorgänge psychischer Natur, sondern physische Vorgänge, deren psychische Folge sich so darstellt, als wäre das durch die Redensarten: ,Trennung der Vorstellung von ihrem Affekt und falsche Verknüpfung des letzteren', Ausgedrückte wirklich geschehen“ (Freud 1940ff., I: 67). Die Nötigung zur ,Bildersprache“ wird auch in Jenseits des Lustprinzips ausgesprochen: „[...] sonst könnten wir die entsprechenden Vorgänge überhaupt nicht beschreiben, ja, würden sie gar nicht wahrgenommen haben“ (Freud 1940ff., XIII: 65). Peter Hofstätter (1949) äußert in einer Arbeit über Platos Kratylos die Ansicht, dass die Psychologie es überhaupt nicht mit einem autochthonen in sich gegliederten Vorstellungsbereich zu tun habe.

Die ganze Eigenart der Psychologie als Wissenschaft liegt darin beschlossen, daß sie ihre ,Gegenstände‘ nicht vorfindet, wie etwa die Physiologie Nerven, Blutgefäße und Sehnen, sondern daß sie jene erst durch die Benennung schafft [...]. [...] dem Namen Denken entspricht nichts von der unterscheidbaren Art eines Dinges, auch nicht etwa eine bestimmte Verlaufsgestalt, [...]. Der Name ,Denken“ hat keinen objektiven Gegenstand, er postuliert im Benennen einen Aspekt, unter dem das Erleben gesehen wird; er deutet also und benennt nicht eigentlich. (Hofstätter 1949: 9)

Warum bediente sich Freud speziell der Analogien aus der Naturwissenschaft und warum übertrug er teilweise den Textsortentypus der Naturwissenschaftssprache des 19. Jahrhunderts in den psychischen Bereich?

Das Phänomen analogischer Übertragung ist kein Sonderfall. Am Anfang des 19. Jahrhunderts kann man beobachten, wie in der deutschen naturphilosophischen Bewegung weit auseinanderliegende Gebiete mit Hilfe des gleichen spekulativen Vokabulars erschlossen werden. Die Sprache von Philosophie, Theologie, Anthropologie und Poesie strahlt in die sich erst herausbildenden Sektoren der Naturwissenschaft aus. Am Ende des 19. Jahrhunderts dominiert umgekehrt die Ausstrahlung der Naturwissenschaften und man beobachtet die Übernahme ihrer Grundbegriffe in die Bereiche der Philosophie und Weltanschauung, Ethik, Sozialtheorie, Politik und Dichtung. Die Lehre Darwins wurde auf sehr unterschiedlichen Gebieten zum Denkmodell, man konnte kurioserweise in der Sprachwissenschaft von einem „Kampf ums Dasein in der Sprache“ (vgl. Sperber 1965: 48; Ullmann 1967: 11, 270) und in der Astronomie von einem „Kampf ums Dasein am Himmel“ (du Prel 1882) sprechen.

Ullmann hat in seinem Aufsatz Laws of Language and Laws of Nature (1943) die Versuche der Junggrammatiker diskutiert, die Lautgesetze den Naturgesetzen 
gleichzustellen und damit die Sprachwissenschaft auf die Höhe der Naturwissenschaft zu erheben. „It is obvious that we are faced with a metaphorical use of the term ,law' [...]. This transfer represents in a nutshell the synthesis of the two factors, or aspirations, which inspired the pioneers of modern linguistics: their veneration of the sound-shift, and their determination to raise and maintain their science on a level with physics and other exact studies." (Ullmann 1943: 328f.) Trotz seiner Skepsis setzt Ullmann dann diesen Versuch fort, wenn er auf dem Gebiet der Synästhesien nach statistisch erfassbaren panchronischen Regularitäten sucht und zu dem Ergebnis kommt, dass der Abstand zwischen den sich an seinem begrenzten Material abzeichnenden statistischen „Sprachgesetzen“ und den statistischen Naturgesetzen der modernen Physik geringer sei als die Kluft zwischen den „Lautgesetzen“ der Junggrammatiker und den Naturgesetzen der klassischen Physik (Ullmann 1943: 338; 1967: 269ff.). In der Chomskyschule haben die Ideen der naturwissenschaftlichen Exaktheit und der mathematischen Charakterisierung der Grundlage menschlicher Spracherzeugung, des ,Mechanismus' bzw. des ,Regelsystems' der Grammatik, den Charakter von Leitprinzipien. Bierwisch behauptete 1966: „Die Regeltypen und ihr Ineinandergreifen [...] können mit mathematischen Mitteln generell und exakt formuliert werden, die Auswirkung unterschiedlicher Annahmen läßt sich genau studieren. Formale Untersuchungen dieser Art haben die Anfänge einer exakten mathematischen Theorie der Sprachkompetenz geschaffen, die nicht in einer bloß äußerlichen Mathematisierung der Linguistik besteht, sondern aus ihrer eigenen Entwicklung ebenso hervorgegangen ist wie die mathematische Formulierung physikalischer oder astronomischer Probleme aus der Entwicklung der Physik und Astronomie.“ (Bierwisch 1966: 116) Eine grundsätzliche Diskussion der ,Natur` unseres Gegenstandes scheint sich anzubahnen.

Um die Ausstrahlung der Naturwissenschaftssprache auf die Psychoanalyse zu erklären, könnte man eine Idee Freuds anführen, die Sperber für die Sprachwissenschaft fruchtbar gemacht hat. Sperber sieht bei der Übertragung von Ausdrücken in eine neue Sphäre eine bestimmte Energiequelle wirksam und findet sie in der Affektstärke, mit dem die Ausgangssphäre bzw. die Zielsphäre behaftet ist. Themenkreise, die im Bewusstsein der Sprachteilhaber mit besonders lebhaften Gefühlen verbunden sind, haben die Tendenz, ihr Vokabular auch in andere Bereiche zu transportieren (Expansion') oder das Vokabular anderer Bereiche an sich zu ziehen (,Attraktion'). ${ }^{10}$ Sperber (1965: 53) denkt dabei im Sinne Freuds an

10 Sperber 1965: Kap. 6-8; in Kap. 10, speziell 67, bemüht sich Sperber um eine naturwissenschaftliche Formulierung der „Gesetzmäßigkeit des Bedeutungswandels“; vgl. Ullmann 1967: 180ff., 230-237. 
affektökonomische Vorgänge, in erster Linie an ein sprachliches Ausdrucksverhalten (das „Streben des Sprechenden, eigene Affekte zu entladen“), dann aber auch an ein berechnendes Appellverhalten (,daß er bewußt auf für den Hörer gefühlsbetonte Vorstellungen anspielt“"). Der Expansionskraft ,älterer` affektbeladener Vorstellungskreise wie Mystik, Alchimie und Belagerungstechnik wären für das Ende des 19. Jahrhunderts sicher die Naturwissenschaften an die Seite zu stellen.

Was Sperber als ,Affektstärke‘ bezeichnet, hat aber zugleich eine soziale Seite; mit ihr müsste der psychologische Aspekt verbunden werden. Die Ausstrahlung der Naturwissenschaften um 1900 auf andere Vorstellungskreise hängt mit ihren Erfolgen, ihrem Sozialprestige zusammen. „Bei Entlehnungen kommt es in erster Linie auf das Prestige an“, schreibt Ullmann. Dabei ist auch an Entlehnungsvorgänge innerhalb der Schichten und Gruppen einer Sprachgemeinschaft (,social borrowing`) zu denken (Ullmann 1967: 94f., 178ff.). Die Teilsprachen sind einem gesellschaftlichen Prestigegefüge zugeordnet, übernommene Wörter evozieren das Prestige ihres ursprünglichen Milieus. Hypothetisch könnte man also Freuds ,naturwissenschaftliche' Sprache auf den Eindruck eines Sozialprestiges zurückführen, oder man könnte sie als wirkungsbewusste Prestigeanleihe erklären, dies um so mehr, als sie für ein als unexakt geltendes Gebiet einen Anschluss an die exakten Wissenschaften herzustellen scheint (vgl. Habermas 1970: 308). Man muss dagegen anführen, dass Freud auf den bildlichen und hypothetischen Charakter seiner ,naturwissenschaftlichen“ Ausdrucksweise nicht selten hinweist. Es wäre trotzdem interessant, welche Rolle für Freud das Modell der Wirkung „des großen Darwin“ (Freud 1940ff., XI: 72) spielte, in welchem Grade er sich Darwins „Umwertung“ der menschlichen Stellung in der Natur (Freud 1940ff., XI: 295) zum Vorbild nahm und sich ihm ansippte und dem naturwissenschaftlich geprägten Zeitgeist in seiner sprachlichen Darstellung bewusst entgegenkam. In den Vorlesungen erklärt er das Sichversprechen zureichend klar aus dem Zusammentreffen zweier Redeabsichten und führt dann den Terminus ,Interferenz‘ ein, der einen physikalischen Vorgang suggeriert und das Bild vom ,Mechanismus des Versprechens‘ stützt (Freud 1940ff., XI: 3. Vorl., 4. Vorl.).

Maßgeblicher für Freuds Verhältnis zu seinem Publikum ist vielleicht die Absicht, zu provozieren. In der pointiert naturwissenschaftlichen Sprache steckt eine Tendenz zur Umwertung geltender Wertvorstellungen (vgl. unten).

Entscheidend für seinen Sprachgebrauch ist aber die Funktion der Erschließung und Darstellung einer Sache. Trier (1934: 196) modifiziert Sperbers Idee der affektiven Suggestion von Vorstellungsbezirken an einer für uns sehr interessanten Stelle nach der sachlichen Seite: Er spricht hier von Wortfeldern „mit bewährter Kraft der Weltaufschließung“, die sich in bestimmten Zeiten über andere Sachbereiche analogisch ausbreiten, sich ,als Mittel anbieten, auch andere Bereiche analogisch zu klären“. „Sie haben Tore zur Welt geöffnet, sie werden auch andere 
Tore öffnen. "Wenn ich recht sehe, haben bei Freud einige Bildfelder die Funktion, umfängliche Bezirke zu erschließen und den Wert von Denkmodellen (vgl. Weinrich 1964b: 23, 26): z.B. das Bild von der Zerlegung der Psyche als eines selbständigen Objekts, das sich auf die Tätigkeit des Forschers und Analytikers bezieht, oder das Bild von der Psyche als eines Energieverteilungsapparates (vgl. Bally 1971: 18ff.; Habermas 1970: 302 ff.). Ob der Sprache dabei eine Eigenständigkeit zufällt, ob es sich bei den Bildfeldern im Sinne Triers um „eigengesetzliche Sinnzentren“ der Sprache handelt, die „vordringlich das Denken der einzelnen lenken“, sei zunächst dahingestellt. Dass diese Ideen der Naturwissenschaft sich „als Mittel anbieten, auch andere Bereiche analogisch zu klären“, leuchtet ein.

Im Hintergrund der Sprache Freuds steht etwas Objektives, das „Dominieren einer Sinnrichtung“ im überindividuellen Bereich (Schwietering 1925: 163), der Zeitgeist also und etwas Subjektives, die persönliche Biographie und Überzeugung: „[...] die exakte naturwissenschaftliche Methodik nämlich, welche er bei seinem Lehrer, dem Physiologen Brücke, kennengelernt hatte, bestimmte zeitlebens sein wissenschaftliches Denken“ (Bally 1971: 11f.). Freud verstand unter ,Psyche` ein nach Prinzipien der Mechanik arbeitendes physisches Objekt, die Psychologie als Naturwissenschaft.

Wenn also ihr Autor das Erfahrene zu ordnen und zu erklären sich anschickt, so sucht er seine Befunde in der Wissenschaft $\mathrm{zu}$ fundieren, die ihm als die einzig zuverlässige erscheint, der Physiologie, die sich ihrerseits auf die Physik als ihre Modellwissenschaft berief. Die Forschung, die den Namen einer Wissenschaft verdiente, mußte eine gegenständliche und die Gegenstände mit Messung erfassende Wissenschaft sein. (Bally 1971: 11f.)

In einem frühen posthum veröffentlichten Entwurf einer Psychologie (1895) versuchte er, sein „physikalistisches Programm“ auszuarbeiten. Er distanzierte sich bald davon, behielt aber das Begriffssystem der „Energieverteilung“ bei, indem er es „mentalistisch“ uminterpretierte (Habermas 1970: 303). „Zeitlebens sehnt sich Freud nach dem Ziel, seine Psychologie, als eine vorläufige Wissenschaft, wieder in der Physiologie aufgehen zu lassen.“ (Bally 1971: 11f.) Seine naturwissenschaftlichen Analogien sind sozusagen ernst gemeint:

Die Mängel unserer Beschreibung würden wahrscheinlich verschwinden, wenn wir anstatt der psychologischen Termini schon die physiologischen oder chemischen einsetzen könnten. Diese gehören zwar auch nur einer Bildersprache an, aber einer uns seit längerer Zeit vertrauten und vielleicht auch einfacheren. (Freud 1940ff., XIII: 65).

Der Versuch, den Typus von Freuds Wissenschaftssprache zu begründen, führt unmittelbar an Fragen der Wertung heran. Im Folgenden soll von Einwänden gegen das Vokabular der Psychoanalyse gesprochen werden. Dabei handelt es sich nicht darum, inhaltliche Auseinandersetzungen zu kommentieren, sondern 
die Wirkung der Sprache Freuds zu beobachten. Mich interessiert die psychologische Wirkung dieser Sprache auf Laien und Fachleute, die Kriterien ihrer Kritik und deren Grundlagen.

\section{Zur öffentlichen und wissenschaftlichen Auseinandersetzung mit dem Vokabular Freuds}

Die Breitenwirkung der Psychoanalyse und die Fähigkeit Freuds, sprechende, treffende Ausdrücke zu prägen, führten dazu, dass von den Spezialausdrücken eine größere Anzahl in ihrer neuen Bedeutung wieder in die Gemeinsprache übernommen wurden. Bei diesem Vorgang scheint es charakteristisch zu sein, dass die Wörter etwas von ihrem Vorstellungsinhalt verlieren und an Bedeutungsumfang gewinnen. Das Fremdwörterbuch von Sanders (1891) enthält die Wörter Traumaticum ,Wundmittel' und traumatisch ,Verwundungen betreffend, darauf bezüglich, davon herrührend‘. Das Vokabular der Psychoanalyse definiert das Substantiv Trauma: „Ereignis im Leben des Subjekts, das definiert wird durch seine Intensität, die Unfähigkeit des Subjekts, adäquat darauf zu antworten, die Erschütterung und die dauerhaft pathogenen Wirkungen, die es in der psychischen Organisation hervorruft“ (Laplanche \& Pontalis 1972: 513). Das Wort ist mit meist abgeblasstem Inhalt gemeinsprachlich (bzw. bildungssprachlich). Man kann heute von einem Faschismustrauma der Deutschen sprechen, es kann aber auch vorkommen, dass Studenten eines Proseminars von dem Trauma der Abschlussklausur sprechen. Die Erweiterung der Anwendungsbereiche wissenschaftlicher Spezialausdrücke erklärt sich sehr leicht aus der in der Regel nur ungenauen Kenntnis ihrer terminologischen Bedeutung. Beispiele für den Übergang psychoanalytischer Termini in die Gemeinsprache oder in den Bildungswortschatz sind weiterhin der Ausdruck abreagieren, den Freud 1893 geprägt hat, das von Bleuler 1910 geprägte und von Freud 1912 übernommene und erweiterte Wort Ambivalenz, die Wörter Psychoanalyse, Analytiker, Aggressivität, Identifizierung, Komplex, psychischer Konflikt, Narzißmus, narzißtisch, Neurose, neurotisch, Todestrieb, Vaterkomplex, das Unbewußte, Unterbewußtsein, Flucht in die Krankheit, Fehlleistung, Verdrängung, Zusammensetzungen mit Zwang - wie Zwangsverhalten. Die Wörter Ödipuskomplex, Regression, Sublimierung, Destruktionstrieb, Fixierung, aufarbeiten, Tagtraum, Überich, freie Assoziation scheinen jedenfalls nicht auf den fachlichen Bereich beschränkt zu sein.

Die stärkste sprachprägende Wirkung hatte Freud natürlich innerhalb seiner Schule, also auf eine kaum übersehbare Flut wissenschaftlicher Literatur. Ein Beispiel sei zitiert: 
Die heute geltende Vorstellung von Primär- und Sekundärvorgang als Entwicklungsstadium berücksichtigt vor allem einen bestimmten Aspekt: die halluzinatorische Wunscherfüllung. Selbst Sandler und Nagera, die so entschieden die Phantasie als Ichfunktion herausgearbeitet und von anderen Triebderivaten abgehoben haben, lassen halluzinatorische Befriedigung als ,grundlegenden Vorläufer der späteren Phantasietätigkeit gelten und beziehen sich dabei auf die Differenz zwischen Primär- und Sekundärvorgang. (Jappe 1971: 52)

Der kurze Text spricht nicht direkt über die Sache, sondern über Literatur über die Sache, also auf einer metasprachlichen Ebene. Die zahlreichen Termini sind nicht wie bei Freud appellativ gebraucht, sondern - z. T. ohne Artikel - als Begriffsnamen in einer Zitierfunktion verwendet. Die Schüler schreiben terminologischer als der Lehrer. Ihre Abhängigkeit drückt sich in einer Übernahme und sorgsamen Beachtung von dessen Termini aus. Im Gegensatz zu dem Lehrer, der von der Sache zum Wort gelangt ist und meist sehr viel unabhängiger von seinen Termini ist, kommen die Schüler häufig vom Wort zur Sache, gehen mit Wörtern um, als hätten sie in ihnen die Sachen, verwenden sie in einer metasprachlichen und Zitierfunktion und bemühen sich um ihre Auslegung. Das Vokabular der Psychoanalyse ist nur ein Beispiel für die Semasiologie der Begriffe Freuds.

Der Übergang von Termini Freuds in die Gemeinsprache und ihre Rezeption in der Fachwelt war kein harmonischer Vorgang. Der Biograph Freuds schreibt: „Es wäre vielleicht einmal eine Studienaufgabe der Geschichte der Wissenschaften, sich mit der Flut der Verleumdungen und Mißverständnisse eingehend zu beschäftigen, in denen sich die explosiven Gefühle Luft machten.“ (Jones 1962: 134) Die Wirkungsgeschichte der Sprache Freuds wäre dabei vermutlich ein für die Sprachwissenschaft lohnender Ausschnitt. Die Sprachgeschichte beruht auf einem Konsensus der Sprachteilhaber in Zustimmung oder Ablehnung, die in der Regel nicht in einer ausdrücklichen Reaktion greifbar sind. In der populären und wissenschaftlichen Kritik an einem neuen Sprachgebrauch manifestieren sich dagegen Wirkungen der Sprache und Sanktionen eines Teils der Sprachgemeinschaft. Es könnte lohnend sein, die Gesichtspunkte, unter denen diese Sprachkritik erfolgt, und die Frage ihrer sprachtheoretischen Grundlagen eingehender auseinanderzusetzen und sich dabei des m. E. sehr interessanten Diskussionsmaterials zu bedienen, das in der Geschichte der Wissenschaft und der Wirkung ihrer Sprache bereitliegt.

Ein besonderes Problem der Beschäftigung mit der Sprachkritik ist, dass die Kritik an der Sprache nahezu unlösbar mit der Kritik an der Sache verquickt ist. Sie meint oft in der Kritik an dem neuen Sprachgebrauch die Kritik an den neuen Auffassungen und Realitäten - unter Umständen auf der Grundlage der längst widerlegten Idee einer spiegelbildlichen, also starren Kongruenz zwischen Sprache und Realität -, oder sie nimmt die Kritik an den Wörtern, z.B. dem ,Jargon der Analytiker oder der ,muttersprachlichen Terminologie‘ der inhaltbezogenen 
Sprachforschung, nur zum Vorwand für die Ablehnung der in diesen Gruppensprachen mitgeteilten Sachen.

Eins scheint sich unzweifelhaft zu ergeben, wenn man sich mit den Einzelbeispielen beschäftigt, die Gegenstand der Sprachkritik gewesen sind: Jede definitive, absolute Äußerung der Sprachkritik zu sprachlichen Einzelerscheinungen ist vom wissenschaftlichen Standpunkt aus sinnlos. Der Sektor, auf dem die Sprachkritik ihre Funktion hat, ist nicht derjenige der Obligatorik. Ihre Maßstäbe haben von Fall zu Fall, von Sprechsituation zu Sprechsituation, einen unterschiedlichen Geltungsgrad, und ihre Urteile lassen sich eigentlich nur in graduellen Kategorien wie ,besser' und ,schlechter', ,angemessener‘ oder ,unangemessener ausdrücken. Die Gründe liegen in dem nur andeutenden bzw. teildefinierenden Charakter der Wörter und der Sachsteuerung unseres Verstehens (vgl. unten), in der Bedeutung des sachlichen und sprachlichen Kontextes für die Erschließung von Wortinhalten, und der grundsätzlich beliebigen Zuordnung eines Ausdrucks zu einem Inhalt. Das Gebiet der Sprachkritik liegt zwischen dem Pol der absoluten Beliebigkeit und dem der obligatorischen Restriktion der Beliebigkeit, zu denen der überwiegende Teil der grammatischen Regeln gehört, und die Geltung ihrer Maßstäbe wird immer wieder durchkreuzt von dem in der Sprache wirksamen Arbitraritätsprinzip, dem ja selbst der obligatorische Regelkodex der Grammatik - wenn auch in längeren Zeitabständen - unterliegt.

Die Kritik an der Sprache der Psychoanalyse kann auf der Bewertung der Sache beruhen, von der sie spricht, und auf den mit ihr verbundenen Gefühlen. Die Sprache Freuds bezog sich zu einem guten Teil auf das seinerzeit verpönte und verschleierte Thema der Sexualität. Man drückte sich in der bildungssprachlich bestimmten Schicht indirekt, andeutend verhüllend, negativ wertend, sakralisierend oder auch gar nicht aus (vgl. Bally 1971: 7). Freud hielt sich nicht an die Konvention der Aussparung, der Hüllwörter und Euphemismen, des Konnotats starker Affekte und Wertungen, sondern drückte sich direkt aus. Die offene Verwendung von Ausdrücken wie Sexualtrieb, analsadistische Stufe, Penisneid, genitale Liebe, phallische Mutter, Harnerotik verletzte Tabus und musste provozieren, noch bevor die Frage ihrer Richtigkeit zur Diskussion stand.

Da seine Darstellungsweise auf der Grenze zwischen schriftstellerischem und wissenschaftlichem Sprachstil liegt und sein wissenschaftliches Begriffssystem aus terminologisierten Ausdrücken der Gemeinsprache besteht, stoßen in der Sprache Freuds zwei Sphären mit entgegengesetzten Konnotaten zusammen: die Sphäre der zumindest tendenziell emotionslosen, wertneutralen, in ihrem Anwendungsbereich und ihrem begrifflichen Inhalt spezifizierten Termini der Wissenschaft und die Sphäre der affektverbundenen, vielfach wertenden, in ihrem Anwendungsbereich und begrifflichem Inhalt weniger spezifizierten Wörter der Umgangssprache. Die Verbindungen genitale Liebe, phallische Mutter drücken die 
metaphorische Spannung zwischen unterschiedlichen Sphären und Wertungen besonders deutlich aus (vgl. oben). Es ist nun charakteristisch für Freuds Gebrauch der umgangssprachlichen Wörter, dass er auf ihr Konnotat keine Rücksicht nimmt, sondern den Wortinhalt, bewusst provozierend, zu neutralisieren versucht. Der Satz „Das kleine Kind ist vor allem schamlos [...]“ (Freud 1940ff., V: 92) meint den Ausdruck wörtlich: ,es ist ohne Scham‘. Mit dem Gebrauch von analsadistisch, kannibalisch, Kloakentheorie verbindet Freud keine Wertung. In der Psychoanalyse wurde z. B. das Verhalten eines Kindes, sich vor anderen nackt zu produzieren, als Exhibitionismus bezeichnet, „obgleich exhibitionistisches Verhalten des Erwachsenen als kriminell gewertet wurde und bekannt war, daß beim Wort Exhibitionismus selbstverständlich die landesübliche Wertung mitschwingen würde“ (Schultz-Hencke 1972: 16). Man beschränkte sich auf den ,wörtlichen“ Inhalt, dehnte den Anwendungsbereich vom Erwachsenen auf das Kind aus und ignorierte die Verwendungssphäre ,Kriminalität' und die mit ihr verbundene Wertung, vielleicht nicht obgleich, sondern weil exhibitionistisches Verhalten des Erwachsenen als kriminell gewertet wurde. Der Ausdruck richtet sich, bewusst provozierend, gegen eine streng trennende Klassifikation der Phänomene auf dem Gebiet des Sexualverhaltens und gegen die landesübliche Wertung. In Freuds Vokabular liegt eine Tendenz zur naturwissenschaftlich orientierten Umwertung der seinerzeitigen Auffassungen von der Sexualität. Dem dient die Projektion des ,wertfreien' biologischen Textsortentypus (z.B. Geschlechtstrieb, Variationen, Abweichungen, Zuchtwahl, Objektwahl, Fortpflanzung) auf dieses Gebiet (vgl. oben). Diese Übertragung mit ihrer Betonung des Sexualtriebes stößt aber in der menschlichen Sphäre auf einen sich als autonom verstehenden sittlichen Vorstellungsbereich, das Wort Sexualtrieb tritt beim Publikum in den Assoziationsbereich von ,erniedrigend, beleidigend, beschämend` und bedeutet einen Affront.

Diese Liebestriebe werden nun in der Psychoanalyse a potiori und von ihrer Herkunft her Sexualtriebe geheißen. Die Mehrzahl der ,Gebildeten“ hat diese Namengebung als Beleidigung empfunden und sich für sie gerächt, indem sie der Psychoanalyse den Vorwurf des ,Pansexualismus‘ entgegenschleuderte. Wer die Sexualität für etwas die menschliche Natur Beschämendes und Erniedrigendes hält, dem steht es ja frei, sich der vornehmeren Ausdrücke Eros und Erotik zu bedienen. (Freud 1940ff., XIII: 99)

Freud verstößt, wenn er Liebestrieb durch Sexualtrieb ersetzt, nicht nur gegen die Konnotate, sondern auch gegen die gemeinsprachliche Bedeutung der Wörter Liebe und Sexualität. In der Zusammensetzung mit Trieb sind die beiden das Grundwort spezifizierenden Bestandteile Liebes- und Sexual- bereits in die biologische Sphäre übertragen, für sich genommen - und als in dem Kompositum teilwirksame Glieder - sind sie keineswegs synonym. Das Wort Liebe hat einen 
weiten vielseitigen Anwendungsbereich und entsprechend ein breites Spektrum von Inhalten. Freud selbst nennt an dieser Stelle ,Geschlechtsliebe‘, ,Selbstliebe‘, ,Eltern-' und ,Kindesliebe', die ,Freundschaft' und die ,allgemeine Menschenliebe‘, die ,Hingebung an konkrete Gegenstände und an abstrakte Ideen` (Freud 1940ff., XIII: 98). Das Wort sexuell bezieht sich nur auf den Genitalbereich. Freud erweitert den Gebrauch dieses Terminus gegen die gemeinsprachliche Konvention. Ist mit dieser Umfangserweiterung nun auch, wie zu erwarten, „die Beschränkung auf einen Teil des Vorstellungsinhalts“ (Paul 1970: 91) verbunden? Freud führt an dieser Stelle einen einschränkenden genetischen Gesichtspunkt ein: a potiori und von ihrer Herkunft her Sexualtriebe‘. An anderer Stelle schränkt er nicht durch Attribute ein, sondern durch ein Synonym: Er nennt das Lutschen des Kindes ein Befriedigungsbedürfnis, das „unabhängig von Ernährung nach Lustgewinn strebt und darum sexuell genannt werden darf und soll“ (Freud 1940ff., XVII: 76). In welcher Weise Freud auch den spezifischen Vorstellungsinhalt und nicht nur den Anwendungsbereich des Wortes sexuell im Einzelnen erweitert haben mag, er hat jedenfalls einen solchen Sprachgebrauch nicht gegen den im öffentlichen Bewusstsein verankerten Begriff deutlich $\mathrm{zu}$ machen und durchzusetzen vermocht.

Schultz-Hencke hat in einer posthum veröffentlichten Arbeit aus dem Jahr 1947 ,die psychoanalytische Begriffswelt‘ einer sehr kritischen Prüfung unterzogen, mit dem Ziel, die Begriffe und Wendungen auf ihre Angemessenheit hin zu prüfen und die verfehlten unter ihnen aus der wissenschaftlichen Diskussion verschwinden zu lassen (Schultz-Hencke 1972: 10f.). Er hält Freuds Begriff des ,Sexuellen', dem er sich ausführlich widmet (Schultz-Hencke 1972: 12-32), für „durchaus fehl am Platze“, er habe an erster Stelle das Bekanntwerden und die Anerkennung der Psychoanalyse verhindert und Verwirrung in die wissenschaftliche Diskussion getragen. Freud habe durch seine Erweiterung des Begriffs provoziert, dass man ihn missverstand. „Es war eine Zumutung an die Leser und Hörer, eine Privatformulierung zu benutzen, die nur mit äußerster Mühe verstanden werden konnte. Erschwerend ist es, wenn auf eine solche Bedeutungserweiterung nicht einmal ausdrücklich hingewiesen wird. In Freuds Fall ist es wahrscheinlich so, daß er einfach unterschätzte, wie völlig unzuverlässig derartig stillschweigende Begriffserweiterungen sind“ (Schultz-Hencke 1972: 12). Außer den genauen Kennern der Tatsachenwelt und Literatur werden alle übrigen „sich an den Alltagssinn des Wortes halten und es so interpretieren, wie sie sich berechtigt fühlen. Dann allerdings ist die Freudsche Lehre ein Pansexualismus, wie er krasser nicht sein kann“ (Schultz-Hencke 1972: 14). Man muss hier hinzufügen, dass die Begriffserweiterung allein nicht der Stein des Anstoßes gewesen ist, sondern dass zumindest in der Öffentlichkeit in erster Linie die Tabuierung der Sexualität, also sein Konnotat einer Erweiterung im Wege stand. 
Etwas Vergleichbares kann man vielleicht insgesamt von der naturwissenschaftlichen Sprache Freuds sagen. Diese Sprache schien einen Anschluss der psychoanalytischen Lehre an die exakten Wissenschaften herzustellen und erhöhte dadurch ihre Wirkung (vgl. Schultz-Hencke 1972: 21). Auf der anderen Seite könnte dieser Anschluss an das materialistisch-mechanistische Weltbild des 19. Jahrhunderts dazu geführt haben, dass der an der Sprache ablesbare Gedanke einer determinierten psychischen Mechanik den hauptsächlichen Stein des Anstoßes bildete und die Diskussion sich gar nicht erst ergab oder sich als weltanschauliche Diskussion am Kern der Entdeckungen Freuds vorbeibewegte (vgl. Borkenau 1956: 65f., 68).

Walter Muschg (1958: 309f.) registriert den Eindruck der Drei Abhandlungen zur Sexualtheorie:

[...] unmenschlich objektive Sprache, ohne Spuren von entgegenkommendem Schmuck, eisklar und schroff wie die Aufstellungen eines Physikers, erbarmungslos ein Arsenal von zerfasernden Begriffen, dieser schneidende Rationalismus geht weit über den durchschnittlichen Habitus der Medizinersprache hinaus [...] spröde, gläserne Unpersönlichkeit kältet den Leser.

Muschg beschreibt hier exakt die Wirkung einer Textsortenübertragung. Gemüthafte, persönliche, menschliche Dinge sind transformiert in den Bereich kühler naturwissenschaftlicher Objektivität und erscheinen dadurch als ins Unmenschliche verfremdet. Der starke Eindruck entsteht dadurch, dass bei dem Thema der menschlichen Liebe - zumal von dem Literaturwissenschaftler - eine gegensätzliche Textsorte erwartet wird, ,wie man sie vom Dichter zu erhalten gewohnt ist“ (vgl. oben), oder zumindest die Erhaltung eines Anteils von dieser Darstellungsweise. Die emotionslose, neutrale, systematische Sachlichkeit Freuds enttäuscht diese Erwartung in extremer Weise und erzeugt dadurch den Eindruck eines umgekehrten Pathos der Affektlosigkeit.

Wenn eine bestimmte Textsortenerwartung in extremer Weise enttäuscht wird, so wird das als ein Verstoß gegen konventionelle Normen Anstoß erregen oder komisch wirken. Einiges in den Abhandlungen ist schwer von einer (gewollt komischen) Travestie zu unterscheiden (Freud 1940ff., V: 49ff.). ,Die psychische Wertschätzung - so stellt Freud staunend fest -, „deren das Sexualobjekt als Wunschziel des Sexualtriebs teilhaftig wird, beschränkt sich in den seltensten Fällen auf dessen Genitalien [...]“ (vgl. Bally 1971: 33).

Neben der innerhalb eines wissenschaftlichen Darstellungsrahmens als sachinadäquat empfundenen Textsorte gibt es die Übertragung der wissenschaftlichen Ausdrucksweise in eine andere Redesituation. Wenn einem ein befreundeter Psychologiestudent sagt „Diese Beethoven-Sonate ist bei mir besetzt“, so hat man den Eindruck, er sei in eine falsche Textsorte geraten. Man würde eher erwarten: „Die 
Sonate ist mir aus bestimmten Gründen sehr wertvoll.“ Der Terminus ,besetzt“ gehört der Sphäre des Unpersönlichen, Objektiven, der ,allgemeinen Interpretation' an. Eine solche Übertragung eines standardisierten Ausdrucks ist allerdings jederzeit möglich und oft der Beginn eines Übergangs in die Gemeinsprache. Als Massenerscheinung sind solche Übertritte von Wörtern aus dem Rahmen ihrer ursprünglichen Verwendungssituation ein lohnender Ausgangspunkt für sprachkritische Überlegungen. Was Karl Korn in der Sprache der verwalteten Welt beschäftigte, war z. T. das Wuchern bestimmter Textsorten.

Die Wissenschaftssprache ist keine für eine private, intime Redesituation geschaffene Textsorte wie etwa die Liebeslyrik. Der Terminus subsummiert Erlebnisinhalte, die im konkreten Fall als sehr persönlich und oft auch als einmalig empfunden werden, unter eine allgemeine Kategorie. In der privaten Gesprächssituation ist die Abneigung gegen Termini technici daher verständlich. Es ist aber charakteristisch für das quasi-intime psychoanalytische Gespräch, dass in ihm der Analytiker gegenüber dem Patienten analysierender Forscher, Therapeut und Anwender von Formeln der Forschung in einer Person ist. Es wäre interessant, wie weit er sich in dieser Situation einem Unbehagen gegen den Texttyp der wissenschaftlichen Objektivierung und Verallgemeinerung gegenübersieht und vor der Aufgabe steht, die ,allgemeinen Interpretationen' $\mathrm{zu}$ individualisieren. Schultz-Hencke (1972: 123; vgl. dazu Bittner 1969: 35, 41) warnt davor, Kurzformeln wie z. B. Vaterhaß im Gespräch zu verwenden.

$\mathrm{Zu}$ den publizistischen Reaktionen auf die Psychoanalyse gehörte anscheinend, dass man ihre Sprache in diffamierender Weise beim Wort nahm. Das Bild der ,Analyse‘, der ,Zerlegung‘, erscheint wieder als das der ,Zersetzung‘. In den Abschnitten Das Echo der Psychoanalyse der Zeitschrift Die psychoanalytische Bewegung (1929-33) finden sich Äußerungen über das „,zweischneidige Chirurgenmesser der Seelenanalyse“ oder die Tendenz zur „Zersetzung des biologisch wohlgelungenen Lebens [...]“ (Die Psychoanalytische Bewegung 1929: 183f.). Als ,zersetzend" steht die Analyse in einer Assoziationsreihe abwertender Synonyma wie „negativ, auflösend, destruktiv“ dem Feld des „positiv Aufbauenden“, und der „Synthese“ gegenüber (Die Psychoanalytische Bewegung 1930: 593, 595; vgl. Der Grosse Brockhaus 1933). Die Vorstellung von der Psychoanalyse wird somit dem populären Bildfeld des das Organische Zersetzenden, Krankheitserregenden assoziiert, das sich im 19. Jahrhundert an den ursprünglich wertneutralen chemischen Fachausdruck ,zersetzen“ heftete, schon in der ersten Jahrhunderthälfte häufig mit dem jüdischen Bevölkerungsteil in Verbindung gebracht wurde, um dann in der NS-Zeit zum stereotypen Schmähwort für die Juden zu werden (vgl. Schäfer 1962).

Lagache schreibt in der Einleitung zum Vokabular der Psychoanalyse: „Die Abneigung gegen die Psychoanalyse kommt zuweilen in sarkastischen Bemerkungen über ihre Sprache zum Ausdruck“ (Laplanche \& Pontalis 1972: 7). Im 
Klappentext heißt es, viele Spezialausdrücke der Psychoanalyse „bleiben dem Laien unverständlich, ja ridikül. Man mokiert sich oft über den ,Jargon“ der Psychoanalytiker [...].“ Auch Muschg (1958: 318) spricht 1930 von einer „nicht immer erfreulichen Handhabung eines neuen Jargons“ in der Freudschule.

Es wurde schon erwähnt, dass die wissenschaftliche Terminologie auch nach ihrer sozialen Seite zu betrachten ist. Sie ist unter diesem Gesichtspunkt eine Zunftsprache, in der ursprünglich fremdsprachliches Material bevorzugt wird, und hat eine gruppenstabilisierende und -abgrenzende Funktion. Etwas Archaisches, die Magie unverständlicher verformelter Sprache, scheint der wissenschaftlichen Autorität zu Hilfe zu kommen und sich in ihr zu überliefern. Die Schüler verhalten sich, auch in der Schule Freuds, zünftiger, terminologischer als ihre Lehrer - teils aus ihrem Verhältnis zu einer Autorität, teils aber auch aus dem Bedürfnis, das Gruppenzugehörigkeitsgefühl und die Prestigewirkung nach außen abzustützen. Die Kritik an dem ,Jargon` beruht zunächst auf dem Widerstand der Laien gegen eine Sondersprache der Fachleute, von deren Verständnis man ausgeschlossen ist. Sie hat sehr ernstzunehmende Aspekte, wenn man sich vor Augen führen lässt, wie sehr sich in den letzten hundert Jahren die Kunstsprache der Wissenschaft und die natürliche Bildungssprache auseinanderentwickelt haben und eine breite, $u$. U. verunklärende Literatur damit beschäftigt ist, die Erkenntnisse der Wissenschaft in die Sprache der Laien zu übersetzen (vgl. Gipper 1956; Portmann 1965). Die Kritik der Laien erscheint als berechtigt, solange die Terminologie des Wissenschaftlers nicht eine sachlich begründete, sondern nur eine sozialabgrenzende Imponierfunktion erfüllt, oder sobald, kein so seltener Fall, der beträchtliche terminologische Apparat in keinem Verhältnis zu den mitgeteilten unkomplizierten oder nichtigen Gedanken und Sachen steht, der Textsortentypus der Wissenschaftssprache also zur Textsortenattrappe wird.

Man könnte hieraus schließen, dass im Hinblick auf die Nichtfachleute eine Wissenschaftssprache vorzuziehen wäre, die sich wie die Psychoanalyse möglichst appellativer, gemeinsprachlicher, vielleicht sogar muttersprachlicher Termini bedient. Die Angelegenheit hat aber - auch unter dem Aspekt der Wissenschaftsrezeption bei der Laienwelt - eine Kehrseite. Karl Bühler hat betont, dass wir sachgesteuert denken, dass unsere Auffassung der Wörter von den mit ihnen bezeichneten Gegenständen bestimmt wird und wir sie weitgehend als Andeutung nehmen. ${ }^{11}$ Darin liegt ein starker Einwand gegen eine Überschätzung der Sprache.

11 Bühler 1965: 171 und 172: „Man ist dort bei den Dingen, von denen gesprochen wird, und läßt die konstruktive oder rekonstruierende Tätigkeit zum guten Teil vo m Gegenstand selbst, den man schon kennt oder soweit er durch den Text bereits angelegt und aufgebaut ist, gesteuert werden [...].“ „Die stoffliche Steuerung des Sprechdenkens ist ein Phänomen, welches mit einigen anderen Tatsachen zusammen den wichtigen Satz zu beweisen gestattet, daß das Andeuten [...] 
Er gilt aber nur solange, als wir uns nicht aufgrund einer bewusst anderen Auffassung der Wörter oder aufgrund unserer Unkenntnis der mit ihnen gemeinten Dinge anders verhalten. Wer von einer Sache nichts weiß, sieht sich veranlasst, sie aus dem Kontext und aus dem Wort zu erschließen. Wem die Bedeutung eines Wortes, z. B. einer neuen Zusammensetzung, nicht vertraut ist, der erschließt sie z. T. aus der Bedeutung seiner ihm vertrauten Bestandteile. Die sog. Volksetymologie und das Etymologisieren der Kinder sind nur extreme Beispiele für das graduell bis in das sprachorientierte Verhalten sich fortsetzende Erschließen neuer Sachen aus den Wörtern. Die sprechenden, u. U. metaphorischen Termini enthalten ja bereits einen Hinweis auf die bezeichneten begrifflichen Inhalte, man versteht sie halbwegs und wird sehr leicht zu der vorschnellen Meinung verführt, sie ganz zu verstehen, die Sache in dem Wort zu haben. Die sprechende Nomenklatur hat den Vorteil, an Bekanntes anzuknüpfen und die Termini merkfähiger zu machen, sie hat aber den Nachteil, ein wortgesteuertes Halbverstehen zu fördern. Und während bei dem Fremdwörterjargon das Gefühl kühler Wissenschaftlichkeit und das Prestige des Fachmännischen konnotiert wird, nimmt ein muttersprachlicher Jargon leicht die umgangssprachlich assoziierten Gefühle und Wertungen in sich auf. Wissenschaftszweige, die mit einer appellativen Terminologie arbeiten, haben möglicherweise eine erhöhte Chance, zu Modeerscheinungen $\mathrm{zu}$ werden, also $\mathrm{zu}$ einer breiten Wirkung und Rezeption ihrer Termini zu gelangen bei nur halber Kenntnis der in dem Vokabular intendierten Sachzusammenhänge. Die Wirkungen Darwins oder Heideggers, vielleicht auch Freuds, ließen sich hier anführen.

Abschließend sei auf Beispiele aus der wissenschaftlichen Auseinandersetzung mit Freuds Sprachgebrauch eingegangen.

Hofstätters Hinweis, dass die Psychologie es nicht mit einem autochthonen, unabhängig von der Benennung gegliederten Vorstellungsbereich zu tun habe (vgl. oben), hat Konsequenzen für seine Bewertung der Sprache: „Im Akt der Benennung erfüllen sich für die Psychologie sehr viel wesentlichere und folgenschwerere Anliegen als für irgendeine andere Disziplin. “12

auch im Bereich der Begriffswörter zu finden ist und zu den Struktureigenheiten der menschlichen Sprache gehört.“

12 Hofstätter 1949: 12. Hofstätter setzt hinzu, „daß die Art und Weise, wie die herrschende Schulpsychologie eines Zeitalters ihre Termini wählt, nicht nur charakteristisch ist für die innere Problematik dieser Epoche, sondern darüber hinaus auch voller Verantwortung sein sollte für die Ausgestaltung des Selbstgesprächs der Menschen dieser Generation.“ Die sprachethische Forderung hat aber bei Hofstätter keine erkennbare Grundlage. Denn wenn der Gegenstandsbereich des Psychologen ein ungegliedertes Kontinuum darstellt, worauf soll sich dann die Verantwortung bei der diesen Bereich gliedernden Benennung stützen? Wenn alles fließt, hat die Sprachkritik keine Grundlage. Das ist die Eingangsthese von Platos Kratylos. 
Gipper hält es für vorteilhaft, wenn als Termini ,Fremdwörter` verwendet werden, weil muttersprachliche, sprechende Ausdrücke „das Denken und Verstehen zu sehr in bestimmte Bahnen lenken“, „weil durch zu wortwörtliche Auslegungen leicht Mißverständnisse verursacht werden. Gerade aus diesen Gründen erweisen sich hierzu Bildungen aus griechisch-lateinischem Sprachmaterial als vorteilhaft: sie wirken verständnisfördernd, ohne eine allzu wörtliche Deutung aufzudrängen, und erleichtern zudem die internationale Kommunizierbarkeit“ (Gipper 1964: 250; 1969: 74). Renz (1971: 307) fordert bei abstrakten,Gegenständen‘, zu denen die psychologischen ja gehören würden, die Verwendung arbiträrer, nur benennender, etiketthafter „Zeichentermini“ an Stelle der „orientierenden.“

Wir haben weiter oben versucht, Gründe für Freuds gemeinsprachliche und vielfach bildhafte Terminologie auseinanderzusetzen. Freud selbst bezeichnet das Problem des orientierenden Terminus mit einem sehr glücklichen, treffenden Ausdruck, wenn er den von Bleuler 1911 geschaffenen Ausdruck ,Schizophrenie“ kritisiert: „Er ist [...] allzu präjudizierlich [...]“ (Freud 1940ff., VIII: 312f.).

Das Problem, dass ein Terminus aufgrund des in dem Wort enthaltenen Hinweises auf seinen Begriffsinhalt ein Phänomen verzeichnet oder verengt, ist in der Geschichte der Wissenschaft immer wieder Anstoß zur Kritik und zur Ersetzung vorhandener Terminologien. Die Kritik braucht nicht zu einer Ersetzung des Terminus zu führen, weil jeder zunächst sprechend gemeinte Ausdruck einer Tendenz zur Lexikalisierung (Idiomatisierung) unterliegt, der durch das Etymon vermittelte Vorstellungsinhalt der Komposita und Metaphern, wie Bühler gezeigt hat, selektiv aufgefasst wird und sehr leicht verblasst und das kontext- und stoffgesteuerte Verstehen die Oberhand gewinnt (Bühler 1965: z.B. 65, 171f., 223f., 233f.; vgl. Fleischer 1969: 10ff.; Gauger 1971: 155f.). Das ist bei dem Namen ,Schizophrenie“ in der Fachwelt eingetreten. Trotzdem konnte er eine wissenschaftliche Auffassung bestimmen, und bei denjenigen, die von der Sache nicht wissen, kann er immer noch als Appellativum benutzt werden, man erläutert: „Schizophrenie heißt Bewußtseinsspaltung [...]“.

Schultz-Henckes Arbeit macht in ausgezeichneter Weise deutlich, wo die Probleme liegen können, wenn Ausdrücke der Gemeinsprache terminologisch verwendet werden. „Wäre Sexualität eine bedeutungslose Nebensache im menschlichen Leben, so würde natürlich niemand so zäh am üblichen Begriffsinhalt festhalten [...]. Da aber die Sexualität einen eminent wichtigen, selbstverständlich interessierenden Bestandteil des Lebens darstellt, hat sich der Begriff zu enormer Stabilität eingefahren“ (Schultz-Hencke 1972: 13). „Es ist völlig aussichtslos, zu erwarten, man werde sich eines Tages dazu bequemen, die Freudsche Begriffsbestimmung zu übernehmen. Dies geht aus dem einfachen Grunde nicht, weil die Menschen dann ein neues Wort erfinden müßten für das, was sie gewöhnlich als Sexualität interessiert“(Schultz-Hencke 1972: 14). Freuds Begriffserweiterung habe 
auch für die Entwicklung der Psychoanalyse als Wissenschaft „,äußerst schwerwiegende Folgen gehabt. Die Vertreter der Freudschen Auffassung haben sich nämlich selbst den Folgen der schiefen Begriffsbildung nicht entziehen können. Sie erlagen immer wieder selbst dem Druck der öffentlichen Meinung: man habe unter sexuell das Übliche zu verstehen“ (Schultz-Hencke 1972: 26). Bei Freuds Erweiterung des Begriffs ,sexuell‘ würde es sich demnach um einen ähnlichen Fall handeln wie bei der sogenannten Umweltlehre des Biologen Jakob von Uexküll. Gipper hat in dem Buch Bausteine zur Sprachinhaltsforschung dargestellt, dass hier eine ganze Theorie fast an einem missverständlichen Sprachgebrauch gescheitert wäre. Uexküll vermochte seinen Begriff von der ,Umwelt‘ der Lebewesen als des mit ihrer jeweiligen leiblich-sinnlichen Ausstattung korrelierenden Erfahrungsraums nicht gegen das allgemeine Verständnis von ,Umwelt‘ als ,die einen Menschen umgebende äußere Lebenswelt‘ durchzusetzen. „Dieser muttersprachliche Inhalt erwies sich als so stark, daß alle Versuche, das Wort als neuen Begriff durchzusetzen, zum Scheitern verurteilt waren. Der gewohnte Inhalt schlug durch [...]. Wer einen neuen Begriff prägt, muß also solche sprachlichen Wirkungen und damit die Durchsetzungschancen sorgfältig abzuschätzen suchen.“(Gipper 1964: 253; 1962: 6. Kap.)

Man muss zwei Sachen auseinanderhalten: die Richtigkeit eines Ausdrucks, d.h. dass für einen gemeinten Vorstellungsinhalt eine der allgemeinen Konvention entsprechende Bezeichnung gewählt wird, und die Wahrheit eines Ausdrucks, d. h. dass der mit dem Ausdruck verbundene Vorstellungsinhalt die gemeinte Sache deckt.

Wenn ich richtig verstehe, kritisiert Schultz-Hencke nicht nur, dass Freud seinen privaten Gebrauch von sexuell gegen eine stabile Sprachkonvention durchsetzen will und ihn dabei nicht ausdrücklich terminologisiert - also auf der Ebene der Sprachrichtigkeit - sondern auch, dass der Ausdruck sexuell, auch in einem erweiterten Sinn, die intendierten Sachverhalte nicht deckt - also auf der Ebene der Wahrheit. „Was Freud unter dem Begriff Sexualität subsummieren wollte, zerfällt in wenigstens drei psychisch völlig originäre, autochthone Gebiete. Diese sind das des Geltungsstrebens, des Besitzstrebens und der Sexualität. Gemeinsam ist ihnen lediglich ihr vital expansiver Charakter“ (Schultz-Hencke 1972: 31, vgl. 14, 17, 26). Die Kritik gilt hier anscheinend nicht einer unzweckmäßigen und missverständlichen Erweiterung eines Wortgebrauchs, sondern einer falschen Platzierung von Tatsachen unter eine Vorstellung, einem unwahren Sprachgebrauch aufgrund eines unzutreffenden Begriffs von der Sache.

Die beiden Gesichtspunkte der Richtigkeit und der Wahrheit sind bei SchultzHencke m. E. vermengt, aber es scheint das Problem gemeinsprachlicher Termini zu sein, dass sie schwer trennbar sind. Die Bezeichnung eines Gegenstandsbereichs mit einem umgangssprachlichen Ausdruck hat zur Folge, dass an diesem Bereich eine bestimmte gängig verbundene Gruppe von Merkmalen isoliert und 
hervorgehoben wird, dass er sozusagen einen ,Spitznamen“ erhält. Dieses Verfahren wird dann besonders kritisch, wenn das Wort an sich einen eng umrissenen Geltungsbereich hat - wie z.B. Sexualität im Vergleich zu Liebe -, in seiner terminologischen Verwendung ein nicht dominantes oder sogar nur nebensächliches Merkmal an einem Phänomenbereich hervorhebt, und nun als vermeintlicher Aufschluss über den ganzen Gegenstandsbereich verkannt wird. Z. B.: „Wenn man über Zwangsneurose las, las man über die Mitschilderung des nicht-sexuellen Analen hinweg, durch das Wort sexuell exquisit auf die oberen erwähnten Koppelungen hingelenkt“ (Schultz-Hencke 1972: 29). Oder: „Das Kind rebelliert überhaupt, es ist motorisch expansiv und in seine Aggressivität fügt sich auch Haß gegen ein konkurrierendes Elternteil ein. Dieser Tatbestand ist nicht schlagwort$\operatorname{artig} \mathrm{zu}$ formulieren. Seine Komplexität und auch seine Feinheit widerspricht dem. Der Ödipuskomplex im streng definierten Sinn findet sich zu spärlich, um als Kernstück aller Neurosen bezeichnet zu werden“ (Schultz-Hencke 1972: 46). In der gleichen Weise erscheint das Wort phallische Stufe als ein Name, der eine Fülle von Tatsachen aus dem 4. und 5. Lebensjahr verengt und verzeichnet (SchultzHencke 1972: 54; vgl. auch 48f. die Bemerkungen zur ,Kastrationsangst').

Der Ausdruck schlagwortartig, Schlagwort wird von Schultz-Hencke (1972: 46, 85) in einem genauen Sinn verwendet: Ein zusammenfassendes Wort hebt an einem heterogenen und komplexen Vorstellungsbereich, der mit starken Affekten verbunden ist, einen $u$. U. nicht einmal dominierenden Aspekt hervor, etikettiert diesen Sachbereich in zuspitzender Weise und schafft einen suggestiven scheinbaren Gesamtüberblick, verabsolutiert also in wirksamer Weise eine Teilwahrheit.

Ob die Darstellung Schultz-Henckes sachlich zutrifft, ist nicht unsere Fragestellung. Das linguistisch Interessante an Freuds Gebrauch des Terminus Sexualität, die Frage seiner sprachlichen „Richtigkeit“ und Zweckmäßigkeit oder die einer in ihm enthaltenen bewussten Provokation, einer Tendenz zur Umwertung einer geltenden Rangordnung, (welche von der „menschlichen Größensucht“ als „empfindlichste Kränkung“ erfahren wird; vgl. Freud 1940ff., XI: 18. Vorl.), bedürfte genauer Textuntersuchungen. Es fällt auf, dass Schultz-Hencke seltener die Termini Freuds selbst als ihre kontextautonome Verwendung in der Freud'schen Schule kritisiert, das Festhalten der Schüler an unzweckmäßigen Begriffen und ihr „Kleben am bloßen Worte des Meisters“ (Schultz-Hencke 1972: 32). Die Abhängigkeit der Schüler, die vom Wort ausgehen und es als Beschreibung eines Phänomenbereichs nehmen, die Verselbständigung der Termini zu einem scheinbaren Aufschluss über die Sachen, ist immer wieder Gegenstand seiner Kritik. „Der Begriff ,phallische Stufe‘ führt die Lernenden in ein Irren, das sich Jahre hinzieht [...]““ (Schultz-Hencke 1972: 56). Der vom Phänomen abgelöste, mit den Termini als ihrem Ersatz umgehende Denkstil wird in Wendungen wie Floskel, pseudowissenschaftliche Phrase, inhaltsleere Formel angegriffen (z.B. 
Schultz-Hencke 1972: 82, 86, 95). Um die Gefahren einer solchen Verwendung der Termini zu vermeiden, schlägt Schultz-Hencke (1972: 42; vgl. auch 18, 85f.) nicht die Verwendung symbolischer Zeichentermini vor, sondern das entgegengesetzte Verfahren einer „exakten und umfänglicheren Beschreibung des Tatbestandes“.

Neben der Frage nach der sachlichen bzw. sprachlichen Richtigkeit der orientierenden Termini Freuds stellt sich die noch schwierigere nach einer Richtigkeit seiner bildhaft orientierenden Ausdrücke und ihrer Wirkung auf das Denken. Schultz-Hencke stellt in verstreuten Bemerkungen zu einigen naturwissenschaftlichen Analogien dar, wie in ihnen eine Hilfsvorstellung reifiziert, das in ihnen enthaltene Vergleichsbild für die Sache selbst genommen wird. Darunter fällt für ihn z.B. der Begriff der ,Libido‘. Der Terminus weise auf keinen neuen Gegenstand hin. Ursprünglich synonym mit ,sexuellem Begehren', beruhe der Begriff in seiner weiteren Ausbildung auf den Spekulationen Freuds über die organischen Korrelate psychischen Geschehens. „So gelangte er zu einem Energiebegriff, der in Analogie zum physikalischen gebildet war [...]. Das Analogische wurde bald außer Acht gelassen und von Freud sogar wohl kaum je gesehen. “ Der Begriff schaffe eine Scheinklärung, „tatsächlich ist lediglich das Beobachtete und Ungeklärte in die Sphäre des Materiellen transportiert worden. Analogien sind aber keine Identitäten“ (Schultz-Hencke 1972: 34). Schultz-Hencke (1972: 35) äußert hier die Ansicht, der Libidobegriff habe letztlich dazu gedient, das als belastend empfundene umgangssprachliche Konnotat des Sexualbegriffs zu vermeiden. „Libido bedeutet gewissermaßen eine rehabilitierte Sexualität.“

Ähnlich beurteilt er den Begriff des ,psychischen Apparats‘, der gerade eben noch als flüchtige Metapher zulässig sei. „Es muß dann sofort zu genauerer Beschreibung dessen übergegangen werden, worum es sich bei dieser Verbalisierung eigentlich handelt. Auf keinen Fall aber darf sich der Untersucher durch seinen Begriff dazu verführen lassen, nun Eigenschaften, die physische Apparate zu haben pflegen, am Psychischen ,abzulesen““ (Schultz-Hencke 1972: 111f.). Schultz-Hencke bezeichnet hier das Problem der Analogie, dass sie dazu führen kann, den Hilfscharakter der gewählten Bildvorstellung nicht mehr wahrzunehmen und bei der weiteren Erschließung der Sache ,im Bild zu bleiben', sich von ihm die Vorstellungen über die Sache vorschreiben und das Denken in bestimmte Bahnen drängen zu lassen. Es ist interessant, wie geschärft bei ihm das Bewusstsein metaphorischen Sprechens ist und wie sehr er bei der Verwendung von Analogien darauf Wert legt, dass sie ausdrücklich bewusst gemacht werden (Schultz-Hencke 1972: 41f., 61, 115f.).

Gustav Bally (1971: 20f.) kritisiert Freuds naturwissenschaftliche Sprache von einem grundsätzlicheren erkenntnistheoretischen Standpunkt aus. Er hält Freuds Verfahren, seine Befunde „in analogistischen Hypothesen mechanistischer Art“ auszudrücken, für „gefährlich“. Die Gefahr liegt in einer Vermengung zweier der 
Sache nach getrennter Sphären, die dazu führt, dass die Psyche als ein monadisch in sich funktionierender Apparat - ohne seine Beziehung zu seinem Gegenüber betrachtet wird. Aus der Sphärenvermengung (vgl. Carnap 1931: 235ff.) folgt eine „,wissenschaftliche‘ Reduktion der ,Person“ [...] auf ein ,Objekt““ (Bally 1971: 30).

Die naheliegende Frage nach der kommunikativen Bedeutung von Haltung, Verhalten, Symptom wird nicht gestellt; der Zug der ,wissenschaftlichen' Verpflichtung zur Zerlegung des ,Gegenstandes“ in seine ,Bausteine‘ und zum erklärenden Begreifen seiner Struktur aus den Elementen läßt Freud nur den Weg vom einzelnen ,Behandlungsobjekt‘ nach einem lediglich hypothetisch zu erfassenden ,Innenleben` frei. Das Problem, daß der Patient als ein Mitmensch, das heißt von seinen (verstellten oder frei spielenden) Kommunikationsmöglichkeiten her verstanden werden könnte, kann sich Freud gar nicht stellen und hat sich ihm nie gestellt. (Bally 1971: 18f.)

Schon ist ein ,Kräftespiel‘ angenommen [...]. In diesem Augenblick wurzeln aber die Begriffe und ihre Hypostasierungen schon nicht mehr in der kategorialen Sphäre, in der das Phänomen zu Hause ist, nämlich der dialogisch-wirhaften Verbundenheit des Sich-Mitteilens und darin Seiner-selbst-Innewerdens; sie sind vielmehr einem System entlehnt, das der mitweltlichen Kommunikation und ihren Voraussetzungen wesensfremd ist. (Bally 1971: 20)

Bally ersetzt hier die Ausdrucksweise Freuds durch die gemeinsprachlichen Termini einer Kommunikationsphilosophie (,kommunikativ', ,Mitmensch', ,dialogisch-wirhafte Verbundenheit', ,Sich-Mitteilen', ,Seiner-selbst-Innewerden'). Es handelt sich um den Versuch, die Lehre Freuds aus ihrer naturwissenschaftlichen Verpuppung (vgl. Trier 1934: 196) zu befreien und sie in eine sphärenadäquate Sprache zu übersetzen. Dieser Ansatz ist von Habermas in Erkenntnis und Interesse und von Lorenzer in dem Buch Sprachzerstörung und Rekonstruktion fortgeführt worden. Habermas (1970: 263) bringt das Problem auf die Formel eines „,szientistischen Selbstmißverständnisses der Psychoanalyse“, deren eigentliche Sphäre er als „Tiefenhermeneutik“ erläutert. Bei beiden rücken an Stelle von Objektstrukturen die Kommunikationsstrukturen ins Zentrum der Beschreibung. Dabei spielt ein der kommunikativen ,Lebenspraxis‘ kurzschlüssig parallelisierter Sprachbegriff eine entscheidende Rolle, ${ }^{13}$ der $\mathrm{m}$. E. eine eingehende Diskussion erfordert. Dieses Problem soll an anderer Stelle weiterverfolgt werden.

13 Lorenzer 1973: 145, 8, legt neuerdings Wert auf „die grundsätzliche Differenzierung zwischen Interaktionsformen und Sprachfiguren“. An anderer Stelle referiert er aber folgendes als „Grundannahmen neuerer Sprachdiskussion“, (von denen er in Sprachzerstörung und Rekonstruktion ausgegangen war): „Aussagen über die Sprachstruktur sind auch Aussagen über die Struktur des Handelns - menschliches Handeln ist symbolisch vermittelt, d.h. es folgt den Regeln, die auch die Sprachstruktur bestimmen.“ „Wenn aber Sprachsymbole als handlungsbestimmend anerkannt werden, dann erscheint Handeln zwangsläufig abgetrennt vom historischen Prozeß.“ (?!) 